\title{
The Generality of a Section of a Curve
}

\author{
Eric Larson
}

\begin{abstract}
Let $f: C \rightarrow \mathbb{P}^{3}$ be a general curve of genus $g$, mapped to $\mathbb{P}^{3}$ via a general linear series of degree $d$; and let $Q$ be a general (and thus smooth) quadric. In this paper, we show that the points of intersection $f(C) \cap Q$ give a general collection of $2 d$ points on $Q$, except for exactly six exceptional cases.

We also prove similar theorems for every other pair $(r, n)$ for which, except for only finitely many pairs $(d, g)$, the intersection of a general curve of genus $g$ mapped to $\mathbb{P}^{r}$ via a general linear series of degree $d$, with a general hypersurface $S$ of degree $n$, is a general collection of $d n$ points on $S$.

As explained in [11, these results play a key role in the author's proof of the Maximal Rank Conjecture [12].
\end{abstract}

\section{Introduction}

If $C$ is a general curve of genus $g$, equipped with a general map $f: C \rightarrow \mathbb{P}^{3}$ of degree $d$, it is natural to ask whether the intersection $f(C) \cap Q$ of its image with a general quadric $Q$ is a general collection of $2 d$ points on $Q$. Interest in this question historically developed as a result of the work of Hirschowitz [6] on the Maximal Rank Conjecture for rational space curves, and the later extension of Ballico and Ellia [3] of this method to nonspecial space curves: The heart of these arguments revolve precisely around understanding the intersection of a general curve with a general quadric. In hopes of both simplifying and extending these results, Ellingsrud and Hirschowitz [4, and later Perrin [13], using the technique of liaison, gave partial results on the generality of this intersection. However, a complete analysis has so far remained conjectural.

The present paper gives the first complete analysis. Both the results and the techniques developed here play a critical role in the author's proof of the Maximal Rank Conjecture [12], as explained in [11].

To state the problem precisely, we make the following definition:

Definition 1.1. We say a stable map $f: C \rightarrow \mathbb{P}^{r}$ from a curve $C$ to $\mathbb{P}^{r}$ (with $r \geq 2$ ) is a Weak Brill-Noether curve (WBN-curve) if it corresponds to a point in a component of $\bar{M}_{g}\left(\mathbb{P}^{r}, d\right)$ which both dominates $\bar{M}_{g}$, and whose generic member is a map from a smooth curve, which is an immersion if $r \geq 3$, and birational onto its image if $r=2$; and which is either nonspecial or nondegenerate. In the latter case, we refer to it as a Brill-Noether curve (BN-curve).

The celebrated Brill-Noether theorem then asserts that BN-curves of degree $d$ and genus $g$ to $\mathbb{P}^{r}$ exist if and only if

$$
\rho(d, g, r):=(r+1) d-r g-r(r+1) \geq 0 .
$$


Moreover, for $\rho(d, g, r) \geq 0$, the parameter space of BN-curves is irreducible. (In particular, it makes sense to talk about a "general BN-curve".)

In this paper, we give a complete answer to the question posed above: For $f: C \rightarrow \mathbb{P}^{3}$ a general BN-curve of degree $d$ and genus $g$ (with, of course, $\rho(d, g, 3) \geq 0$ ), we show the intersection $f(C) \cap Q$ is a general collection of $2 d$ points on $Q$ except in exactly six cases. Furthermore, in these six cases, we compute precisely what the intersection is.

A natural generalization of this problem is to study the intersection of a general BN-curve $f: C \rightarrow \mathbb{P}^{r}$ (for $r \geq 2$ ) with a hypersurface $H$ of degree $n \geq 1$ : In particular, we ask when this intersection consists of a general collection of $d n$ points on $H$ (in all but finitely many cases).

For $r=2$, the divisor $f(C) \cap H$ on $H$ is linearly equivalent to $\mathcal{O}_{H}(d)$; in particular, it can only be general if $H$ is rational, i.e. if $n=1$ or $n=2$. In general, we note that in order for the intersection to be general, it is evidently necessary for

$$
(r+1) d-(r-3) g \sim(r+1) d-(r-3)(g-1)=\operatorname{dim} \bar{M}_{g}\left(\mathbb{P}^{r}, d\right)^{\circ} \geq(r-1) \cdot d n .
$$

(Here $\bar{M}_{g}\left(\mathbb{P}^{r}, d\right)^{\circ}$ denotes the component of $\bar{M}_{g}\left(\mathbb{P}^{r}, d\right)$ corresponding to the BN-curves, and $A \sim B$ denotes that $A$ differs from $B$ by a quantity bounded by a function of $r$ alone.) If the genus of $C$ is as large as possible (subject to the constraint that $\rho(d, g, r) \geq 0$ ), i.e. if

$$
g \sim \frac{r+1}{r} \cdot d
$$

then the intersection can only be general when

$$
(r+1) \cdot d-(r-3) \cdot\left(\frac{r+1}{r} \cdot d\right) \gtrsim(r-1) n \cdot d
$$

or equivalently if

$$
(r+1)-(r-3) \cdot \frac{r+1}{r} \geq(r-1) n \quad \Leftrightarrow \quad n \leq \frac{3 r+3}{r^{2}-r}
$$

For $r=3$, this implies $n=1$ or $n=2$; for $r=4$, this implies $n=1$; and for $r \geq 5$, this is impossible.

To summarize, there are only five pairs $(r, n)$ where this intersection could be, with the exception of finitely many $(d, g)$ pairs, a collection of $d n$ general points on $H$ : The intersection of a plane curve with a line, the intersection of a plane curve with a conic, the intersection of a space curve with a quadric, the intersection of a space curve with a plane, and the intersection of a curve to $\mathbb{P}^{4}$ with a hyperplane. Our three main theorems (five counting the first two cases which are trivial) give a complete description of this intersection in these cases:

Theorem 1.2. Let $f: C \rightarrow \mathbb{P}^{2}$ be a general BN-curve of degree $d$ and genus $g$. Then the intersection $f(C) \cap Q$, of $C$ with a general conic $Q$, consists of a general collection of $2 d$ points on $Q$.

Theorem 1.3. Let $f: C \rightarrow \mathbb{P}^{2}$ be a general BN-curve of degree $d$ and genus $g$. Then the intersection $f(C) \cap L$, of $C$ with a general line L, consists of a general collection of $d$ points on $L$. 
Theorem 1.4. Let $f: C \rightarrow \mathbb{P}^{3}$ be a general BN-curve of degree $d$ and genus $g$. Then the intersection $f(C) \cap Q$, of $C$ with a general quadric $Q$, consists of a general collection of $2 d$ points on $Q$, unless

$$
(d, g) \in\{(4,1),(5,2),(6,2),(6,4),(7,5),(8,6)\} .
$$

And conversely, in the above cases, we may describe the intersection $f(C) \cap Q \subset Q \simeq \mathbb{P}^{1} \times \mathbb{P}^{1}$ in terms of the intrinsic geometry of $Q \simeq \mathbb{P}^{1} \times \mathbb{P}^{1}$ as follows:

- If $(d, g)=(4,1)$, then $f(C) \cap Q$ is the intersection of two general curves of bidegree $(2,2)$.

- If $(d, g)=(5,2)$, then $f(C) \cap Q$ is a general collection of 10 points on a curve of bidegree $(2,2)$.

- If $(d, g)=(6,2)$, then $f(C) \cap Q$ is a general collection of 12 points $p_{1}, \ldots, p_{12}$ lying on a curve $D$ which satisfy:

- The curve D is of bidegree (3,3) (and so is in particular of arithmetic genus 4).

- The curve D has two nodes (and so is in particular of geometric genus 2).

- The divisors $\mathcal{O}_{D}(2,2)$ and $p_{1}+\cdots+p_{12}$ are linearly equivalent when pulled back to the normalization of $D$.

- If $(d, g)=(6,4)$, then $f(C) \cap Q$ is the intersection of two general curves of bidegrees $(2,2)$ and $(3,3)$ respectively.

- If $(d, g)=(7,5)$, then $f(C) \cap Q$ is a general collection of 14 points $p_{1}, \ldots, p_{14}$ lying on a curve $D$ which satisfy:

- The curve $D$ is of bidegree $(3,3)$.

- The divisor $p_{1}+\cdots+p_{14}-\mathcal{O}_{D}(2,2)$ on $D$ is effective.

- If $(d, g)=(8,6)$, then $f(C) \cap Q$ is a general collection of 16 points on a curve of bidegree $(3,3)$.

In particular, the above descriptions show $f(C) \cap Q$ is not a general collection of $2 d$ points on $Q$.

Theorem 1.5. Let $f: C \rightarrow \mathbb{P}^{3}$ be a general BN-curve of degree $d$ and genus $g$. Then the intersection $f(C) \cap H$, of $C$ with a general plane $H$, consists of a general collection of d points on $H$, unless

$$
(d, g)=(6,4)
$$

And conversely, for $(d, g)=(6,4)$, the intersection $f(C) \cap H$ is a general collection of 6 points on a conic in $H \simeq \mathbb{P}^{2}$; in particular, it is not a general collection of $d=6$ points. 
Theorem 1.6. Let $f: C \rightarrow \mathbb{P}^{4}$ be a general BN-curve of degree $d$ and genus $g$. Then the intersection $f(C) \cap H$, of $C$ with a general hyperplane $H$, consists of a general collection of $d$ points on $H$, unless

$$
(d, g) \in\{(8,5),(9,6),(10,7)\} .
$$

And conversely, in the above cases, we may describe the intersection $f(C) \cap H \subset H \simeq \mathbb{P}^{3}$ in terms of the intrinsic geometry of $H \simeq \mathbb{P}^{3}$ as follows:

- If $(d, g)=(8,5)$, then $f(C) \cap H$ is the intersection of three general quadrics.

- If $(d, g)=(9,6)$, then $f(C) \cap H$ is a general collection of 9 points on a curve $E \subset \mathbb{P}^{3}$ of degree 4 and genus 1.

- If $(d, g)=(8,5)$, then $f(C) \cap H$ is a general collection of 10 points on a quadric.

The above theorems can be proven by studying the normal bundle of the general BN-curve $f: C \rightarrow \mathbb{P}^{r}$ : For any hypersurface $S$ of degree $n$, and unramified map $f: C \rightarrow \mathbb{P}^{r}$ dimensionally transverse to $S$, basic deformation theory implies that the map

$$
f \mapsto(f(C) \cap S)
$$

(from the corresponding Kontsevich space of stable maps, to the corresponding symmetric power of $S$ ) is smooth at $[f]$ if and only if

$$
H^{1}\left(N_{f}(-n)\right)=0 .
$$

Here, $N_{f}(-n)=N_{f} \otimes f^{*} \mathcal{O}_{\mathbb{P}^{r}}(-n)$ denotes the twist of the normal bundle $N_{f}$ of the map $f: C \rightarrow \mathbb{P}^{r}$; this is the vector bundle on the domain $C$ of $f$ defined via

$$
N_{f}=\operatorname{ker}\left(f^{*} \Omega_{\mathbb{P} r} \rightarrow \Omega_{C}\right)^{\vee} .
$$

Since a map between reduced irreducible varieties is dominant if and only if it is generically smooth, the map $f \mapsto(f(C) \cap S)$ is therefore dominant if and only if $H^{1}\left(N_{f}(-n)\right)=0$ for $[f]$ general.

This last condition being visibly open, our problem is thus to prove the existence of an unramified BN-curve $f: C \rightarrow \mathbb{P}^{r}$ of specified degree and genus, for which $H^{1}\left(N_{f}(-n)\right)=0$. For this, we will use a variety of techniques, most crucially specialization to a map from a reducible curve $X \cup_{\Gamma} Y \rightarrow \mathbb{P}^{r}$.

We begin, in Section 2, by giving several tools for studying the normal bundle of a map from a reducible curve. Then in Section 3, we review results on the closely-related interpolation problem (c.f. 2]). In Section [4, we review results about when certain maps from reducible curves, of the type we shall use, are BN-curves. Using these techniques, we then concentrate our attention in Section 5 on maps from reducible curves $X \cup_{\Gamma} Y \rightarrow \mathbb{P}^{r}$ where $Y$ is a line or canonical curve. Consideration of these curves enables us to make an inductive argument that reduces our main theorems to finite casework.

This finite casework is then taken care of in three steps: First, in Sections 6 8, we again use degeneration to a map from a reducible curve, considering the special case when $Y \rightarrow \mathbb{P}^{r}$ factors through a hyperplane. Second, in Section 9, we specialize to immersions of smooth 
curves contained in Del Pezzo surfaces, and study the normal bundle of our curve using the normal bundle exact sequence for a curve in a surface. Lastly, in Section 10 we use the geometry of the cubic scroll in $\mathbb{P}^{4}$ to construct an example of an immersion of a smooth curve $f: C \hookrightarrow \mathbb{P}^{3}$ of degree 5 and genus 1 with $H^{1}\left(N_{f}(-2)\right)=0$.

Finally, in Section 11, we examine each of the cases in our above theorems where the intersection is not general. In each of these cases, we work out precisely what the intersection is (and show that it is not general).

\section{Conventions}

In this paper we make the following conventions:

- We work over an algebraically closed field of characteristic zero.

- A curve shall refer to a nodal curve, which is assumed to be connected unless otherwise specified.

\section{Acknowledgements}

The author would like to thank Joe Harris for his guidance throughout this research. The author would also like to thank Gavril Farkas, Isabel Vogt, and members of the Harvard and MIT mathematics departments for helpful conversations; and to acknowledge the generous support both of the Fannie and John Hertz Foundation, and of the Department of Defense (NDSEG fellowship).

\section{Normal Bundles of Maps from Reducible Curves}

In order to describe the normal bundle of a map from a reducible curve, it will be helpful to introduce some notions concerning modifications of vector bundles. The interested reader is encouraged to consult [2] (sections 2, 3, and 5), where these notions are developed in full; we include here only a brief summary, which will suffice for our purposes.

Definition 2.1. If $f: X \rightarrow \mathbb{P}^{r}$ is a map from a scheme $X$ to $\mathbb{P}^{r}$, and $p \in X$ is a point, we write $\left[T_{p} C\right] \subset \mathbb{P}^{r}$ for the projective realization of the tangent space - i.e. for the linear subspace $L \subset \mathbb{P}^{r}$ containing $f(p)$ and satisfying $T_{f(p)} L=f_{*}\left(T_{p} C\right)$.

Definition 2.2. Let $\Lambda \subset \mathbb{P}^{r}$ be a linear subspace, and $f: C \rightarrow \mathbb{P}^{r}$ be an unramified map from a curve. Write $U_{f, \Lambda} \subset C$ for the open subset of points $p \in C$ so that the projective realization of the tangent space $\left[T_{p} C\right]$ does not meet $\Lambda$. Suppose that $U_{f, \Lambda}$ is nonempty, and contains the singular locus of $C$. Define

$$
\left.\left.N_{f \rightarrow \Lambda}\right|_{U_{f, \Lambda}} \subset N_{f}\right|_{U_{f, \Lambda}}
$$

as the kernel of the differential of the projection from $\Lambda$ (which is regular on a neighborhood of $f\left(U_{f, \Lambda}\right)$ ). We then let $N_{f \rightarrow \Lambda}$ be the unique extension of $\left.N_{f \rightarrow \Lambda}\right|_{U_{f, \Lambda}}$ to a sub-vector-bundle (i.e. a subsheaf with locally free quotient) of $N_{f}$ on $C$. For a more thorough discussion of this construction (written for $f$ an immersion but which readily generalizes), see Section 5 of [2]. 
Definition 2.3. Given a subbundle $\mathcal{F} \subset \mathcal{E}$ of a vector bundle on a scheme $X$, and a Cartier divisor $D$ on $X$, we define

$$
\mathcal{E}[D \rightarrow \mathcal{F}]
$$

as the kernel of the natural map

$$
\left.\mathcal{E} \rightarrow(\mathcal{E} / \mathcal{F})\right|_{D}
$$

Note that $\mathcal{E}[D \rightarrow \mathcal{F}]$ is naturally isomorphic to $\mathcal{E}$ on $X \backslash D$. Additionally, note that $\mathcal{E}[D \rightarrow \mathcal{F}]$ depends only on $\left.\mathcal{F}\right|_{D}$. For a more thorough discussion of this construction, see Sections 2 and 3 of $[2]$.

Definition 2.4. Given a subspace $\Lambda \subset \mathbb{P}^{r}$, an unramified map $f: C \rightarrow \mathbb{P}^{r}$ from a curve, and a Cartier divisor $D$ on $C$, we define

$$
N_{f}[D \rightarrow \Lambda]:=N_{f}\left[D \rightarrow N_{f \rightarrow \Lambda}\right]
$$

We note that these constructions can be iterated on a smooth curve: Given subbundles $\mathcal{F}_{1}, \mathcal{F}_{2} \subset \mathcal{E}$ of a vector bundle on a smooth curve, there is a unique subbundle $\mathcal{F}_{2}^{\prime} \subset \mathcal{E}\left[D_{1} \rightarrow \mathcal{F}_{1}\right]$ which agrees with $\mathcal{F}_{2}$ away from $D_{1}$ (c.f. Proposition 3.1 of [2]). We may then define:

$$
\mathcal{E}\left[D_{1} \rightarrow \mathcal{F}_{1}\right]\left[D_{2} \rightarrow \mathcal{F}_{2}\right]:=\mathcal{E}\left[D_{1} \rightarrow \mathcal{F}_{1}\right]\left[D_{2} \rightarrow \mathcal{F}_{2}^{\prime}\right]
$$

Basic properties of this construction (as well as precise conditions when such iterated modifications make sense for higher-dimensional varieties) are investigated in [2] (Sections 2 and 3). For example, we have natural isomorphisms $\mathcal{E}\left[D_{1} \rightarrow \mathcal{F}_{1}\right]\left[D_{2} \rightarrow \mathcal{F}_{2}\right] \simeq \mathcal{E}\left[D_{2} \rightarrow \mathcal{F}_{2}\right]\left[D_{1} \rightarrow \mathcal{F}_{1}\right]$ in several cases, including when $\mathcal{F}_{1} \subseteq \mathcal{F}_{2}$.

Using these constructions, we may give a partial characterization of the normal bundle $N_{f}$ of an unramified map from a reducible curve $f: X \cup_{\Gamma} Y \rightarrow \mathbb{P}^{r}$ :

Proposition 2.5 (Hartshorne-Hirschowitz). Let $f: X \cup_{\Gamma} Y \rightarrow \mathbb{P}^{r}$ be an unramified map from a reducible curve. Write $\Gamma=\left\{p_{1}, p_{2}, \ldots, p_{n}\right\}$, and for each $i$ let $q_{i} \neq f\left(p_{i}\right)$ be a point on the projective realization $\left[T_{p_{i}} Y\right]$ of the tangent space to $Y$ at $p_{i}$. Then we have

$$
\left.N_{f}\right|_{X}=N_{\left.f\right|_{X}}(\Gamma)\left[p_{1} \rightarrow q_{1}\right]\left[p_{2} \rightarrow q_{2}\right] \cdots\left[p_{n} \rightarrow q_{n}\right] .
$$

Proof. This is Corollary 3.2 of [5], re-expressed in the above language. (Hartshorne and Hirschowitz states this only for $r=3$ and $f$ an immersion; but the argument they give works for $r$ arbitrary.)

Our basic strategy to study the normal bundle of an unramified map from a reducible curve $f: C \cup_{\Gamma} D \rightarrow \mathbb{P}^{r}$ is given by the following lemma:

Lemma 2.6. Let $f: C \cup_{\Gamma} D \rightarrow \mathbb{P}^{r}$ be an unramified map from a reducible curve, and let $E$ and $F$ be divisors supported on $C \backslash \Gamma$ and $D \backslash \Gamma$ respectively. Suppose that the natural map

$$
\alpha: H^{0}\left(N_{\left.f\right|_{D}}(-F)\right) \rightarrow \bigoplus_{p \in \Gamma}\left(\frac{T_{p}\left(\mathbb{P}^{r}\right)}{f_{*}\left(T_{p}\left(C \cup_{\Gamma} D\right)\right)}\right)
$$


is surjective (respectively injective), and that

$$
\begin{gathered}
H^{1}\left(\left.N_{f}\right|_{D}(-F)\right)=0 \quad\left(\text { respectively } H^{0}\left(\left.N_{f}\right|_{D}(-F)\right)=H^{0}\left(N_{\left.f\right|_{D}}(-F)\right)\right) \\
H^{1}\left(N_{\left.f\right|_{C}}(-E)\right)=0 \quad\left(\text { respectively } H^{0}\left(N_{\left.f\right|_{C}}(-E)\right)=0\right) .
\end{gathered}
$$

Then we have

$$
H^{1}\left(N_{f}(-E-F)\right)=0 \quad\left(\text { respectively } H^{0}\left(N_{f}(-E-F)\right)=0\right) .
$$

Proof. Write $\mathcal{K}$ for the sheaf supported along $\Gamma$ whose stalk at $p \in \Gamma$ is the quotient of tangent spaces:

$$
\mathcal{K}_{p}=\frac{T_{p}\left(\mathbb{P}^{r}\right)}{f_{*}\left(T_{p}\left(C \cup_{\Gamma} D\right)\right)} .
$$

Additionally, write $\mathcal{N}$ for the (not locally-free) subsheaf of $N_{f}$ "corresponding to deformations which do not smooth the nodes $\Gamma$ "; or in symbols, as the kernel of the natural map

$$
N_{f} \rightarrow T_{\Gamma}^{1}
$$

where $T^{1}$ is the Lichtenbaum-Schlessinger $T^{1}$-functor. We have the following exact sequences of sheaves:

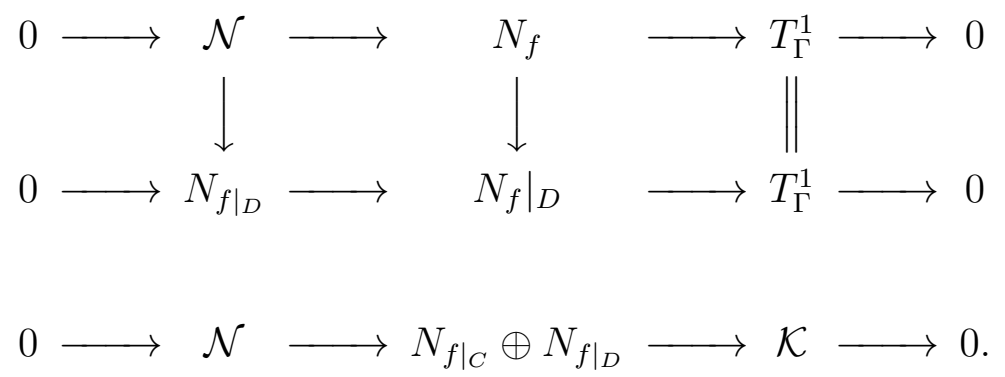

The first of sequence above is just the definition of $\mathcal{N}$. Restriction of the first sequence to $D$ yields the second sequence (we have $\left.\mathcal{N}\right|_{D} \simeq N_{\left.f\right|_{D}}$ ); the map between them being of course the restriction map. The final sequence expresses $\mathcal{N}$ as the gluing of $\left.\mathcal{N}\right|_{C} \simeq N_{\left.f\right|_{C}}$ to $\left.\mathcal{N}\right|_{D} \simeq N_{\left.f\right|_{D}}$ along $\left.\mathcal{N}\right|_{\Gamma} \simeq \mathcal{K}$.

Twisting everything in sight by $-E-F$, we obtain new sequences:

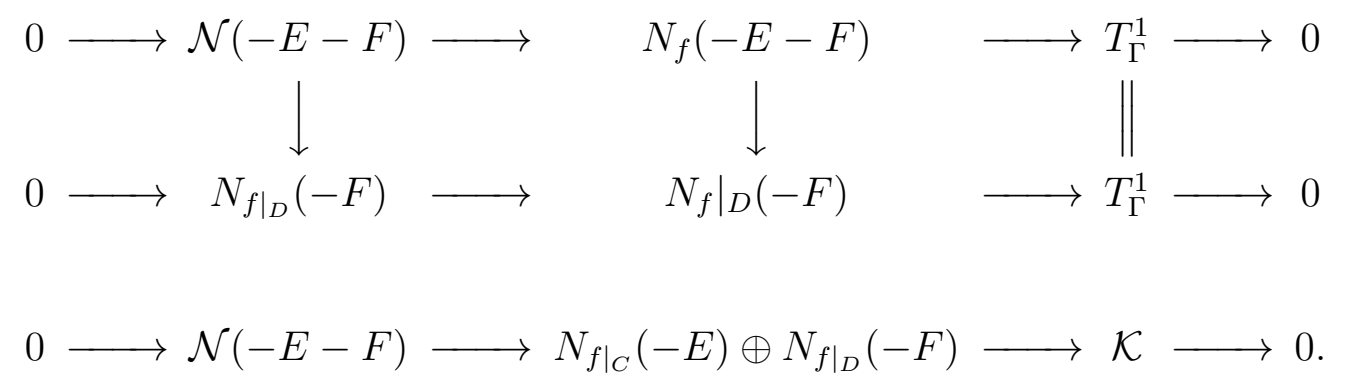

The commutativity of the rightmost square in the first diagram implies that the image of $H^{0}\left(N_{f}(-E-F)\right) \rightarrow H^{0}\left(T_{\Gamma}^{1}\right)$ is contained in the image of $H^{0}\left(\left.N_{f}\right|_{D}(-F)\right) \rightarrow H^{0}\left(T_{\Gamma}^{1}\right)$. Consequently, we have

$$
\begin{aligned}
\operatorname{dim} H^{0}\left(N_{f}(-E-F)\right) & =\operatorname{dim} H^{0}(\mathcal{N}(-E-F))+\operatorname{dim} \operatorname{Im}\left(H^{0}\left(N_{f}(-E-F)\right) \rightarrow H^{0}\left(T_{\Gamma}^{1}\right)\right) \\
& \leq \operatorname{dim} H^{0}(\mathcal{N}(-E-F))+\operatorname{dim} \operatorname{Im}\left(H^{0}\left(\left.N_{f}\right|_{D}(-F)\right) \rightarrow H^{0}\left(T_{\Gamma}^{1}\right)\right) \\
& =\operatorname{dim} H^{0}(\mathcal{N}(-E-F))+\operatorname{dim} H^{0}\left(\left.N_{f}\right|_{D}(-F)\right)-\operatorname{dim} H^{0}\left(N_{\left.f\right|_{D}}(-F)\right) .
\end{aligned}
$$


Next, our assumption that $H^{0}\left(N_{\left.f\right|_{D}}(-F)\right) \rightarrow H^{0}(\mathcal{K})$ is surjective (respectively our assumptions that $H^{0}\left(N_{\left.f\right|_{C}}(-E)\right)=0$ and $H^{0}\left(N_{\left.f\right|_{D}}(-F)\right) \rightarrow H^{0}(\mathcal{K})$ is injective) implies in particular that $H^{0}\left(N_{\left.f\right|_{C}}(-E) \oplus N_{\left.f\right|_{D}}(-F)\right) \rightarrow H^{0}(\mathcal{K})$ is surjective (respectively injective).

In the "respectively" case, this yields $H^{0}(\mathcal{N}(-E-F))=0$, which combined with (1) and our assumption that $H^{0}\left(\left.N_{f}\right|_{D}(-F)\right)=H^{0}\left(N_{\left.f\right|_{D}}(-F)\right)$ implies $H^{0}\left(N_{f}(-E-F)\right)=0$ as desired. In the other case, we have a bit more work to do; the surjectivity of $H^{0}\left(N_{\left.f\right|_{D}}(-F)\right) \rightarrow H^{0}(\mathcal{K})$ yields

$$
\operatorname{dim} H^{0}(\mathcal{N}(-E-F))=\operatorname{dim} H^{0}\left(N_{\left.f\right|_{C}}(-E) \oplus N_{\left.f\right|_{D}}(-F)\right)-\operatorname{dim} H^{0}(\mathcal{K}) ;
$$

or upon rearrangement,

$$
\begin{aligned}
\operatorname{dim} H^{0}(\mathcal{N}(-E-F))-\operatorname{dim} H^{0}\left(N_{\left.f\right|_{D}}(-F)\right) & =\operatorname{dim} H^{0}\left(N_{\left.f\right|_{C}}(-E)\right)-\operatorname{dim} H^{0}(\mathcal{K}) \\
& =\chi\left(N_{\left.f\right|_{C}}(-E)\right)-\chi(\mathcal{K}) .
\end{aligned}
$$

(For the last equality, $\operatorname{dim} H^{0}\left(N_{\left.f\right|_{C}}(-E)\right)=\chi\left(N_{\left.f\right|_{C}}(-E)\right)+\operatorname{dim} H^{1}\left(N_{\left.f\right|_{C}}(-E)\right)=\chi\left(N_{\left.f\right|_{C}}(-E)\right)$ because $H^{1}\left(N_{\left.f\right|_{C}}(-E)\right)=0$ by assumption. Additionally, $\operatorname{dim} H^{0}(\mathcal{K})=\chi(\mathcal{K})$ because $\mathcal{K}$ is punctual.)

Substituting this into (1), and noting that $\operatorname{dim} H^{0}\left(\left.N_{f}\right|_{D}(-F)\right)=\chi\left(\left.N_{f}\right|_{D}(-F)\right)$ because $H^{1}\left(\left.N_{f}\right|_{D}(-F)\right)=0$ by assumption, we obtain:

$$
\begin{aligned}
\operatorname{dim} H^{0}\left(N_{f}(-E-F)\right) & \leq \operatorname{dim} H^{0}\left(\left.N_{f}\right|_{D}(-F)\right)+\operatorname{dim} H^{0}(\mathcal{N}(-E-F))-\operatorname{dim} H^{0}\left(N_{\left.f\right|_{D}}(-F)\right) \\
& =\chi\left(\left.N_{f}\right|_{D}(-F)\right)+\chi\left(N_{\left.f\right|_{C}}(-E)\right)-\chi(\mathcal{K}) \\
& =\chi\left(\left.N_{f}\right|_{D}(-F)\right)+\chi\left(\left.N_{f}\right|_{C}(-E-\Gamma)\right) \\
& =\chi\left(N_{f}(-E-F)\right) .
\end{aligned}
$$

For the final two equalities, we have used the exact sequences of sheaves

$$
\begin{gathered}
\left.0 \rightarrow N_{f}\right|_{C}(-E-\Gamma) \rightarrow N_{\left.f\right|_{C}}(-E) \rightarrow \mathcal{K} \rightarrow 0 \\
\left.\left.0 \rightarrow N_{f}\right|_{C}(-E-\Gamma) \rightarrow N_{f}(-E-F) \rightarrow N_{f}\right|_{D}(-F) \rightarrow 0 ;
\end{gathered}
$$

which are just twists by $-E-F$ of the exact sequences:

$$
\begin{gathered}
\left.0 \rightarrow N_{f}\right|_{C}(-\Gamma) \rightarrow N_{\left.f\right|_{C}} \rightarrow \mathcal{K} \rightarrow 0 \\
\left.\left.0 \rightarrow N_{f}\right|_{C}(-\Gamma) \rightarrow N_{f} \rightarrow N_{f}\right|_{D} \rightarrow 0 .
\end{gathered}
$$

To finish, we note that, by (2),

$$
\operatorname{dim} H^{1}\left(N_{f}(-E-F)\right)=\operatorname{dim} H^{0}\left(N_{f}(-E-F)\right)-\chi\left(N_{f}(-E-F)\right) \leq 0,
$$

and so $H^{1}\left(N_{f}(-E-F)\right)=0$ as desired.

In the case where $\left.f\right|_{D}$ factors through a hyperplane, the hypotheses of Lemma 2.6] become easier to check:

Lemma 2.7. Let $f: C \cup_{\Gamma} D \rightarrow \mathbb{P}^{r}$ be an unramified map from a reducible curve, such that $\left.f\right|_{D}$ factors as a composition of $f_{D}: D \rightarrow H$ with the inclusion of a hyperplane $\iota: H \subset \mathbb{P}^{r}$, while $\left.f\right|_{C}$ 
is transverse to $H$ along $\Gamma$. Let $E$ and $F$ be divisors supported on $C \backslash \Gamma$ and $D \backslash \Gamma$ respectively. Suppose that, for some $i \in\{0,1\}$,

$$
H^{i}\left(N_{f_{D}}(-\Gamma-F)\right)=H^{i}\left(\mathcal{O}_{D}(1)(\Gamma-F)\right)=H^{i}\left(N_{\left.f\right|_{C}}(-E)\right)=0 .
$$

Then we have

$$
H^{i}\left(N_{f}(-E-F)\right)=0 .
$$

Proof. If $i=0$, we note that $H^{0}\left(\mathcal{O}_{D}(1)(\Gamma-F)\right)=0$ implies $H^{0}\left(\mathcal{O}_{D}(1)(-F)\right)=0$. In particular, using the exact sequences

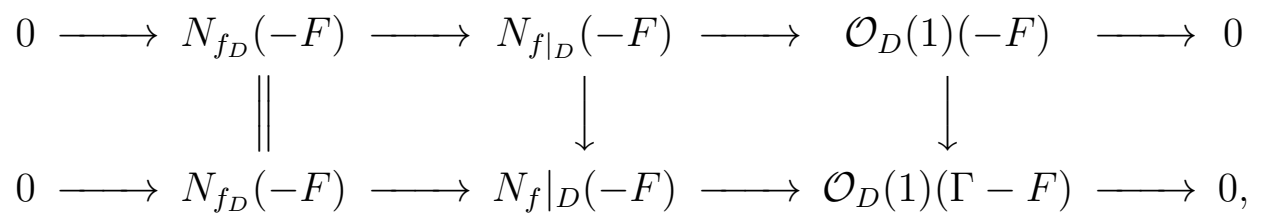

we conclude from the first sequence that $H^{0}\left(N_{f_{D}}(-F)\right) \rightarrow H^{0}\left(N_{\left.f\right|_{D}}(-F)\right)$ is an isomorphism, and from the 5-lemma applied to the corresponding map between long exact sequences that $H^{0}\left(N_{\left.f\right|_{D}}(-F)\right)=H^{0}\left(\left.N_{f}\right|_{D}(-F)\right)$.

Similarly, when $i=1$, we note that $H^{1}\left(N_{f_{D}}(-\Gamma-F)\right)=0$ implies $H^{1}\left(N_{f_{D}}(-F)\right)=0$; we thus conclude from the second sequence that $H^{1}\left(\left.N_{f}\right|_{D}(-F)\right)=0$.

It thus remains to check that the map $\alpha$ in Lemma 2.6 is injective if $i=0$ and surjective if $i=1$. For this we use the commutative diagram

$$
\begin{gathered}
\left.H^{0}\left(N_{f_{D}}(-F)\right) \stackrel{\beta}{\longrightarrow} N_{f_{D}}\right|_{\Gamma} \simeq \bigoplus_{p \in \Gamma}\left(\frac{T_{p} H}{f_{*}\left(T_{p} D\right)}\right) \\
\downarrow \downarrow \\
H^{0}\left(N_{\left.f\right|_{D}}(-F)\right) \stackrel{\alpha}{\longrightarrow} \bigoplus_{p \in \Gamma}\left(\frac{T_{p}\left(\mathbb{P}^{r}\right)}{f_{*}\left(T_{p}\left(C \cup_{\Gamma} D\right)\right)}\right) .
\end{gathered}
$$

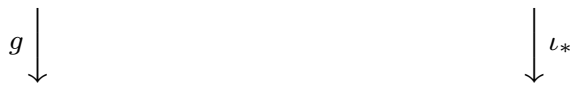

Since $\left.f\right|_{C}$ is transverse to $H$ along $\Gamma$, the map $\iota_{*}$ above is an isomorphism. In particular, since $g$ is an isomorphism when $i=0$, it suffices to check that $\beta$ is injective if $i=0$ and surjective if $i=1$. But using the exact sequence

$$
\left.0 \rightarrow N_{f_{D}}(-\Gamma-F) \rightarrow N_{f_{D}}(-F) \rightarrow N_{f_{D}}\right|_{\Gamma} \rightarrow 0,
$$

this follows from our assumption that $H^{i}\left(N_{f_{D}}(-\Gamma-F)\right)=0$.

\section{Interpolation}

If we generalize $N_{f}(-n)$ to $N_{f}(-D)$, where $D$ is a general effective divisor, we get the problem of "interpolation." Geometrically, this corresponds to asking if there is a curve of degree $d$ and genus $g$ which passes through a collection of points which are general in $\mathbb{P}^{r}$ (as opposed to general in a hypersurface $S$ ). This condition is analogous in some sense to the conditions of semistability and section-semistability (see Section 3 of [1]), as well as to the Raynaud condition (property $\star$ of [14]); although we shall not make use of these analogies here. 
Definition 3.1. We say a vector bundle $\mathcal{E}$ on a curve $C$ satisfies interpolation if it is nonspecial, and for a general effective divisor $D$ of any degree,

$$
H^{0}(\mathcal{E}(-D))=0 \quad \text { or } \quad H^{1}(\mathcal{E}(-D))=0 .
$$

We have the following results on interpolation from [2]. To rephrase them in our current language, note that if $f: C \rightarrow \mathbb{P}^{r}$ is a general BN-curve for $r \geq 3$, then $f$ is an immersion, so $N_{f}$ coincides with the normal bundle $N_{f(C) / \mathbb{P}^{r}}$ of the image. Note also that, from Brill-Noether theory, a general BN-curve $f: C \rightarrow \mathbb{P}^{r}$ of degree $d$ and genus $g$ is nonspecial (i.e. satisfies $\left.H^{1}\left(f^{*} \mathcal{O}_{\mathbb{P}^{r}}(1)\right)=0\right)$ if and only if $d \geq g+r$.

Proposition 3.2 (Theorem 1.3 of [2]). Let $f: C \rightarrow \mathbb{P}^{r}$ (for $r \geq 3$ ) be a general BN-curve of degree $d$ and genus $g$, where

$$
d \geq g+r
$$

Then $N_{f}$ satisfies interpolation, unless

$$
(d, g, r) \in\{(5,2,3),(6,2,4),(7,2,5)\} .
$$

Proposition 3.3 (Proposition 4.12 of [2]). Let $\mathcal{E}$ be a vector bundle on a curve $C$, and $D$ be a divisor on $C$. If $\mathcal{E}$ satisfies interpolation and

$$
\chi(\mathcal{E}(-D)) \geq(\operatorname{rk} \mathcal{E}) \cdot(\text { genus } C)
$$

then $\mathcal{E}(-D)$ satisfies interpolation. In particular,

$$
H^{1}(\mathcal{E}(-D))=0
$$

Lemma 3.4. Let $f: C \rightarrow \mathbb{P}^{r}$ (for $\left.r \in\{3,4,5\}\right)$ be a general $B N$-curve of degree $r+2$ and genus 2. Then $H^{1}\left(N_{f}(-1)\right)=0$.

Proof. We will show that there exists an immersion $C \hookrightarrow \mathbb{P}^{r}$, which is a BN-curve of degree $r+2$ and genus 2 , and whose image meets a hyperplane $H$ transversely in a general collection of $r+2$ points. For this, we first find a rational normal curve $R \subset H$ passing through $r+2$ general points, which is possible by Corollary 1.4 of [2]. This rational normal curve is then the hyperplane section of some rational surface scroll $S \subset \mathbb{P}^{r}$ (and we can freely choose the projective equivalence class of $S$ ).

It thus suffices to prove that there exists a smooth curve $C \subset S$, for which $C \subset S \subset \mathbb{P}^{r}$ is a BN-curve of degree $r+2$ and genus 2 , such that $C \cap(H \cap S)$ a set of $r+2$ general points on $H \cap S$; or alternatively such that the map

$$
C \mapsto(C \cap(H \cap S)),
$$

from the Hilbert scheme of curves on $S$, to the Hilbert scheme of points on $H \cap S$, is smooth at $[C]$; this in turn would follow from $H^{1}\left(N_{C / S}(-1)\right)=0$.

But by Corollary 13.3 of [2], the general BN-curve $C^{\prime} \subset \mathbb{P}^{r}$ (which is an immersion since $r \geq 3$ ) of degree $r+2$ and genus 2 in $\mathbb{P}^{r}$ is contained in some rational surface scroll $S^{\prime}$, and satisfies $\chi\left(N_{C^{\prime} / S^{\prime}}\right)=11$. Since we can choose $S$ projectively equivalent to $S^{\prime}$, we may thus find a BN-curve $C \subset S$ of degree $r+2$ and genus 2 with $\chi\left(N_{C / S}\right)=11$. But then,

$$
\chi\left(N_{C / S}(-1)\right)=11-d \geq g \quad \Rightarrow \quad H^{1}\left(N_{C / S}(-1)\right)=0 .
$$


Combining these results, we obtain:

Lemma 3.5. Let $f: C \rightarrow \mathbb{P}^{r}$ (for $r \geq 3$ ) be a general BN-curve of degree $d$ and genus $g$. Suppose that $d \geq g+r$.

- If $r=3$ and $g=0$, then $H^{1}\left(N_{f}(-2)\right)=0$. In fact, $N_{f}(-2)$ satisfies interpolation.

- If $r=3$, then $H^{1}\left(N_{f}(-1)\right)=0$. In fact, $N_{f}(-1)$ satisfies interpolation except when $(d, g)=(5,2)$.

- If $r=4$ and $d \geq 2 g$, then $H^{1}\left(N_{f}(-1)\right)=0$. In fact, $N_{f}(-1)$ satisfies interpolation except when $(d, g)=(6,2)$.

Proof. When $(d, g, r) \in\{(5,2,3),(6,2,4)\}$, the desired result follows from Lemma 3.4, Otherwise, from Propositions 3.2 , we know that $N_{f}$ satisfies interpolation. Hence, the desired conclusion follows by applying Proposition 3.3. If $r=3$, then

$$
\begin{aligned}
& \chi\left(N_{f}(-1)\right)=2 d \geq 2 g=(r-1) g \\
& \chi\left(N_{f}(-2)\right)=0=(r-1) g ;
\end{aligned}
$$

and if $r=4$ and $d \geq 2 g$, then

$$
\chi\left(N_{f}(-1)\right)=2 d-g+1 \geq 3 g=(r-1) g .
$$

Lemma 3.6. Suppose $f: C \cup_{u} L \rightarrow \mathbb{P}^{3}$ is an unramified map from a reducible curve, with $L \simeq \mathbb{P}^{1}$, and $u$ a single point, and $\left.f\right|_{L}$ of degree 1 . Write $v \neq f(u)$ for some other point on $f(L)$. If

$$
H^{1}\left(N_{\left.f\right|_{C}}(-2)(u)[2 u \rightarrow v]\right)=0
$$

then we have

$$
H^{1}\left(N_{f}(-2)\right)=0 .
$$

Proof. We apply Lemma 8.5 of [2] (which is stated for $f$ an immersion, in which case $N_{f}=N_{C \cup L}$ and $N_{\left.f\right|_{C}}=N_{C}$, but the same proof works whenever $f$ is unramified); we take $N_{C}^{\prime}=N_{\left.f\right|_{C}}(-2)$ and $\Lambda_{1}=\Lambda_{2}=\emptyset$. This implies $N_{f}(-2)$ satisfies interpolation (c.f. Definition 3.1) provided that $N_{\left.f\right|_{C}}(-2)(u)[u \rightarrow v][u \rightarrow v]$ satisfies interpolation. But we have

$$
\chi\left(N_{f}(-2)\right)=\chi\left(N_{\left.f\right|_{C}}(-2)(u)[u \rightarrow v][u \rightarrow v]\right)=0
$$

so both of these interpolation statements are equivalent to the vanishing of $H^{1}$. That is, we have $H^{1}\left(N_{f}(-2)\right)=0$, provided that

$$
H^{1}\left(N_{\left.f\right|_{C}}(-2)(u)[u \rightarrow v][u \rightarrow v]\right)=H^{1}\left(N_{\left.f\right|_{C}}(-2)(u)[2 u \rightarrow v]\right)=0,
$$

as desired.

We finish this section with the following proposition, which immediately implies Theorems 1.2 and 1.3 . 
Proposition 3.7. Let $f: C \rightarrow \mathbb{P}^{2}$ be a curve. Then $N_{f}(-2)$ satisfies interpolation. In particular $H^{1}\left(N_{f}(-2)\right)=H^{1}\left(N_{f}(-1)\right)=0$.

Proof. By adjunction,

$$
N_{f} \simeq K_{C} \otimes f^{*} K_{\mathbb{P}^{3}}^{-1} \simeq K_{f}(3) \quad \Rightarrow \quad N_{f}(-2) \simeq K_{C}(1)
$$

By Serre duality,

$$
H^{1}\left(K_{C}(1)\right) \simeq H^{0}\left(\mathcal{O}_{C}(-1)\right)^{\vee}=0
$$

which since $K_{C}(1)$ is a line bundle implies it satisfies interpolation.

\section{Reducible BN-Curves}

Definition 4.1. Let $\Gamma \subset \mathbb{P}^{r}$ be a finite set of $n$ points. A pair $\left(f: C \rightarrow \mathbb{P}^{r}, \Delta \subset C_{\mathrm{sm}}\right)$, where $C$ is a curve, $f$ is map from $C$ to $\mathbb{P}^{r}$, and $\Delta$ is a subset of $n$ points on the smooth locus $C_{\mathrm{sm}}$, shall be called a marked curve (respectively marked $B N$-curve, respectively marked $W B N$-curve) passing through $\Gamma$ if $f: C \rightarrow \mathbb{P}^{r}$ is a map from a curve (respectively a BN-curve, respectively a WBN-curve) and $f(\Delta)=\Gamma$.

Given a marked curve $\left(f: C \rightarrow \mathbb{P}^{r}, \Delta\right)$ passing through $\Gamma$, we realize $\Gamma$ as a subset of $C$ via $\Gamma \simeq \Delta \subset C$.

For $p \in \Gamma$, we then define the tangent line $T_{p}(f, \Gamma)$ at $p$ to be the unique line $\ell \subset \mathbb{P}^{r}$ through $p$ with $T_{p} \ell=f_{*} T_{p} C$.

Let $\Gamma \subset \mathbb{P}^{r}$ be a finite set of $n$ general points, and $\left(f_{i}: C_{i} \rightarrow \mathbb{P}^{r}, \Gamma_{i}\right)$ be marked WBN-curves passing through $\Gamma$. We then write $C_{1} \cup_{\Gamma} C_{2}$ for the curve obtained from $C_{1}$ and $C_{2}$ by gluing $\Gamma_{1}$ to $\Gamma_{2}$ via the isomorphism $\Gamma_{1} \simeq \Gamma \simeq \Gamma_{2}$. The maps $f_{i}$ give rise to a map $f: C_{1} \cup_{\Gamma} C_{2} \rightarrow \mathbb{P}^{r}$ from a reducible curve. Then we have the following result:

Proposition 4.2 (Theorem 1.3 of [10]). Suppose that, for at least one $i \in\{1,2\}$, we have

$$
(r+1) d_{i}-r g_{i}+r \geq r n .
$$

Then $f: C_{1} \cup_{\Gamma} C_{2} \rightarrow \mathbb{P}^{r}$ is a WBN-curve.

Proposition 4.3. In Proposition 4.2, suppose that $\left[f_{1}, \Gamma_{1}\right]$ is general in some component of the space of marked WBN-curves passing through $\Gamma$, and that $H^{1}\left(N_{f_{2}}\right)=0$. Then $H^{1}\left(N_{f}\right)=0$.

Proof. This follows from combining Lemmas 3.2 and 3.4 of [10].

The following lemmas give information about the spaces of marked BN-curves passing through small numbers of points.

Lemma 4.4. Let $\Gamma \subset \mathbb{P}^{r}$ be a general set of $n \leq r+2$ points, and $d$ and $g$ be integers with $\rho(d, g, r) \geq 0$. Then the space of marked BN-curves of degree $d$ and genus $g$ to $\mathbb{P}^{r}$ passing through $\Gamma$ is irreducible. 
Proof. First note that, since $n \leq r+2$, any $n$ points in linear general position are related by an automorphism of $\mathbb{P}^{r}$. Fix some ordering on $\Gamma$.

The space of BN-curves of degree $d$ and genus $g$ is irreducible, and the source of the generic $\mathrm{BN}$-curve is irreducible; consequently the space of such BN-curves with an ordered collection of $n$ marked points, and the open subset thereof where the images of the marked points are in linear general position, is irreducible. It follows that the space of such marked curves endowed with an automorphism bringing the images of the ordered marked points to $\Gamma$ (respecting our fixed ordering on $\Gamma$ ) is also irreducible. But by applying the automorphism to the curve and forgetting the order of the marked points, this latter space dominates the space of such BNcurves passing through $\Gamma$; the space of such BN-curves passing through $\Gamma$ is thus irreducible.

Lemma 4.5. Let $\Gamma \subset \mathbb{P}^{r}$ be a general set of $n \leq r+2$ points, and $\left\{\ell_{p}: p \in \Gamma\right\}$ be a set of lines with $p \in \ell_{p}$.

Then the general marked rational normal curve passing through $\Gamma$ has tangent lines at each point $p \in \Gamma$ distinct from $\ell_{p}$.

Proof. Since the intersection of dense opens is a dense open, it suffices to show the general marked rational normal curve $\left(f: C \rightarrow \mathbb{P}^{r}, \Delta\right)$ passing through $\Gamma$ has tangent line at $p$ distinct from $\ell_{p}$ for any one $p \in \Gamma$.

For this we consider the map, from the space of such marked rational normal curves, to the space of lines through $p$, which associates to the curve its tangent line at $p$. Basic deformation theory implies this map is smooth (and thus nonconstant) at $(f, \Delta)$ so long as $H^{1}\left(N_{f}(-\Delta)(-q)\right)=0$, where $q \in \Delta$ is the point sent to $p$ under $f$, which follows from combining Propositions 3.2 and 3.3 .

Lemma 4.6. A general $B N$-curve $f: C \rightarrow \mathbb{P}^{r}$ can be specialized to an unramified map from a reducible curve $f^{\circ}: X \cup_{\Gamma} Y \rightarrow \mathbb{P}^{r}$, where $\left.f^{\circ}\right|_{X}$ is a rational normal curve.

Proof. Write $d$ and $g$ for the degree and genus of $f$. We first note it suffices to produce a marked WBN-curve $\left(f_{2}^{\circ}: Y \rightarrow \mathbb{P}^{r}, \Gamma_{2}\right)$ of degree $d-r$ and genus $g^{\prime} \geq g-r-1$, passing through a set $\Gamma$ of $g+1-g^{\prime}$ general points. Indeed, $g+1-g^{\prime} \leq g+1-(g-r-1)=r+2$ by assumption; by Lemma 4.5, there is a marked rational normal curve $\left(f_{1}^{\circ}: X \rightarrow \mathbb{P}^{r}, \Gamma_{1}\right)$ passing through $\Gamma$, whose tangent lines at $\Gamma$ are distinct from the tangent lines of $\left(f_{2}^{\circ}, \Gamma_{2}\right)$ at $\Gamma$. Then $f^{\circ}: X \cup_{\Gamma} Y \rightarrow \mathbb{P}^{r}$ is unramified (as promised by our conventions) and gives the required specialization by Proposition 4.2 .

It remains to construct $\left(f_{2}^{\circ}: Y \rightarrow \mathbb{P}^{r}, \Gamma_{2}\right)$. If $g \leq r$, then we note that since $d$ and $g$ are integers,

$$
d \geq d-\frac{\rho(d, g, r)}{r+1}=g+r-\frac{g}{r+1} \quad \Rightarrow \quad d \geq g+r \quad \Leftrightarrow \quad g+1 \leq(d-r)+1 .
$$

Consequently, by inspection, there is a marked rational curve $\left(f_{2}^{\circ}: Y \rightarrow \mathbb{P}^{r}, \Gamma_{2}\right)$ of degree $d-r$ passing through a set $\Gamma$ of $g+1$ general points.

On the other hand, if $g \geq r+1$, then we note that $\rho(d-r, g-r-1, r)=(r+1)(d-r)-r(g-r-1)-r(r+1)=(r+1) d-r g-r(r+1)=\rho(d, g, r) \geq 0$.

We may therefore let $\left(f_{2}^{\circ}: Y \rightarrow \mathbb{P}^{r}, \Gamma_{2}\right)$ be a marked BN-curve of degree $d-r$ and genus $g-r-1$ passing through a set $\Gamma$ of $r+2$ general points. 
Lemma 4.7. Let $\Gamma \subset \mathbb{P}^{r}$ be a general set of $n \leq r+2$ points, $\left\{\ell_{p}: p \in \Gamma\right\}$ be a set of lines with $p \in \ell_{p}$, and $d$ and $g$ be integers with $\rho(d, g, r) \geq 0$.

Then the general marked $B N$-curve $\left(f: C \rightarrow \mathbb{P}^{r}, \Delta\right)$ of degree $d$ and genus $g$ passing through $\Gamma$ has tangent lines at every $p \in \Gamma$ which are distinct from $\ell_{p}$.

Proof. By Lemma 4.6, we may specialize $f: C \rightarrow \mathbb{P}^{r}$ to $f^{\circ}: X \cup_{\Gamma} Y \rightarrow \mathbb{P}^{r}$ where $\left.f^{\circ}\right|_{X}$ is a rational normal curve. Specializing the marked points $\Delta$ to lie on $X$ (which can be done since a marked rational normal curve can pass through $n \leq r+2$ general points by Proposition 3.2), it suffices to consider the case when $f$ is a rational normal curve. But this case was already considered in Lemma 4.5.

Lemma 4.8. Lemma 4.6 remains true even if we instead ask $\left.f^{\circ}\right|_{X}$ to be an arbitrary nondegenerate specialization of a rational normal curve.

Proof. We employ the construction used in the proof of Lemma 4.6, but flipping the order in which we construct $X$ and $Y$ : First we fix $\left(f_{1}^{\circ}: X \rightarrow \mathbb{P}^{r}, \Gamma_{1}\right)$; then we construct $\left(f_{2}^{\circ}: Y \rightarrow \mathbb{P}^{r}, \Gamma_{2}\right)$ passing through $\Gamma$, whose tangent lines at $\Gamma$ are distinct from the tangent lines of $\left(f_{1}^{\circ}, \Gamma_{1}\right)$ at $\Gamma$ thanks to Lemma 4.7.

\section{Inductive Arguments}

Let $f: C \cup_{u} L \rightarrow \mathbb{P}^{r}$ be an unramified map from a reducible curve, with $L \simeq \mathbb{P}^{1}$, and $u$ a single point, and $\left.f\right|_{L}$ of degree 1. By Proposition 4.2, these curves are BN-curves.

Lemma 5.1. If $H^{1}\left(N_{\left.f\right|_{C}}(-1)\right)=0$, then $H^{1}\left(N_{f}(-1)\right)=0$.

Proof. This is immediate from Lemma 2.6 (taking $D=L$ ).

Lemma 5.2. If $H^{1}\left(N_{\left.f\right|_{C}}(-2)\right)=0$, and $f$ is a general map of the above type extending $\left.f\right|_{C}$, then $H^{1}\left(N_{f}(-2)\right)=0$.

Proof. By Lemma 3.6, it suffices to prove that for $(u, v) \in C \times \mathbb{P}^{3}$ general,

$$
H^{1}\left(N_{\left.f\right|_{C}}(-2)(u)[2 u \rightarrow v]\right)=0 .
$$

Since $H^{1}\left(N_{\left.f\right|_{C}}(-2)\right)=0$, we also have $H^{1}\left(N_{\left.f\right|_{C}}(-2)(u)\right)=0$; in particular, Riemann-Roch implies

$$
\begin{aligned}
\operatorname{dim} H^{0}\left(N_{\left.f\right|_{C}}(-2)(u)\right) & =\chi\left(N_{\left.f\right|_{C}}(-2)(u)\right)=2 \\
\operatorname{dim} H^{0}\left(N_{\left.f\right|_{C}}(-2)\right) & =\chi\left(N_{\left.f\right|_{C}}(-2)\right)=0 .
\end{aligned}
$$

The above dimension estimates imply there is a unique section $s \in \mathbb{P} H^{0}\left(N_{\left.f\right|_{C}}(-2)(u)\right)$ with $\left.\left.s\right|_{u} \in N_{\left.f\right|_{C} \rightarrow v}\right|_{u}$; it remains to show that for $(u, v)$ general, $\left\langle\left. s\right|_{2 u}\right\rangle \neq\left. N_{\left.f\right|_{C} \rightarrow v}\right|_{2 u}$. For this, it suffices to verify that if $v_{1}$ and $v_{2}$ are points with $\left\{v_{1}, v_{2}, f(2 u)\right\}$ coplanar - but neither $\left\{v_{1}, v_{2}, f(u)\right\}$, nor $\left\{v_{1}, f(2 u)\right\}$, nor $\left\{v_{2}, f(2 u)\right\}$ collinear; and $\left\{v_{1}, v_{2}, f(3 u)\right\}$ not coplanar then $\left.N_{\left.f\right|_{C} \rightarrow v_{1}}\right|_{2 u} \neq\left. N_{\left.f\right|_{C} \rightarrow v_{2}}\right|_{2 u}$. 
To show this, we choose a local coordinate $t$ on $C$, and coordinates on an appropriate affine open $\mathbb{A}^{3} \subset \mathbb{P}^{3}$, so that:

$$
\begin{aligned}
f(t) & =\left(t, t^{2}+O\left(t^{3}\right), O\left(t^{3}\right)\right) \\
v_{1} & =(1,0,1) \\
v_{2} & =(-1,0,1) .
\end{aligned}
$$

It remains to check that the vectors $f(t)-v_{1}, f(t)-v_{2}$, and $\frac{d}{d t} f(t)$ are linearly independent at first order in $t$. That is, we want to check that the determinant

$$
\left|\begin{array}{ccc}
t-1 & t^{2}+O\left(t^{3}\right) & O\left(t^{3}\right)-1 \\
t+1 & t^{2}+O\left(t^{3}\right) & O\left(t^{3}\right)-1 \\
1 & 2 t+O\left(t^{2}\right) & O\left(t^{2}\right)
\end{array}\right| \not \equiv 0 \quad \bmod t^{2}
$$

Or, reducing the entries of the left-hand side modulo $t^{2}$, that

$$
-4 t=\left|\begin{array}{ccc}
t-1 & 0 & -1 \\
t+1 & 0 & -1 \\
1 & 2 t & 0
\end{array}\right| \not \equiv 0 \bmod t^{2}
$$

which is clear.

Lemma 5.3. Let $\Gamma \subset \mathbb{P}^{3}$ be a set of 5 general points, $\left(f_{1}: C \rightarrow \mathbb{P}^{3}, \Gamma_{1}\right)$ be a general marked $B N$-curve passing through $\Gamma$, and $\left(f_{2}: D \rightarrow \mathbb{P}^{3}, \Gamma_{2}\right)$ be a general marked canonical curve passing through $\Gamma$. If $H^{1}\left(N_{f_{1}}(-2)\right)=0$, then $f: C \cup_{\Gamma} D \rightarrow \mathbb{P}^{r}$ satisfies $H^{1}\left(N_{f}(-2)\right)=0$.

Remark 5.4. By Lemma 4.4, it makes sense to speak of a "general marked BN-curve (respectively general marked canonical curve) passing through $\Gamma$ "; by Lemma 4.7, the resulting curve $f$ is unramified.

Proof. By Lemma 2.6, our problem reduces to showing that the natural map

$$
H^{0}\left(N_{f_{2}}(-2)\right) \rightarrow \bigoplus_{p \in \Gamma}\left(\frac{T_{p}\left(\mathbb{P}^{r}\right)}{f_{*}\left(T_{p}\left(C \cup_{\Gamma} D\right)\right)}\right)
$$

is surjective, and that

$$
H^{1}\left(\left.N_{f}\right|_{D}(-2)\right)=0 .
$$

These conditions both being open, we may invoke Lemma 4.6 to specialize $\left(f_{1}: C \rightarrow \mathbb{P}^{3}, \Gamma_{1}\right)$ to a marked BN-curve with reducible source $\left(f_{1}^{\circ}: C_{1} \cup_{\Delta} C_{2} \rightarrow \mathbb{P}^{3}, \Gamma_{1}^{\circ}\right)$, with $\left.f_{1}^{\circ}\right|_{C_{1}}$ a rational normal curve and $\Gamma_{1}^{\circ} \subset C_{1}$. It thus suffices to prove the above statements in the case when $f_{1}=f_{1}^{\circ}$ is a rational normal curve.

For this, we first observe that $f(C) \cap f(D)=\Gamma$ : Since there is a unique rational normal curve through any 6 points, and a 1-dimensional family of possible sixth points on $D$ once $D$ and $\Gamma$ are fixed - but there is a 2-dimensional family of rational normal curves through 5 points in linear general position - dimension counting shows $f_{1}(C)$ and $f_{2}(D)$ cannot meet at a sixth point for $\left(\left[f_{1}, \Gamma_{1}\right],\left[f_{2}, \Gamma_{2}\right]\right)$ general. In particular, $f$ is an immersion. 
Next, we observe that $f(D)$ is contained in a 5-dimensional space of cubics. Since it is one linear condition, for a cubic that vanishes on $f(D)$, to be tangent to $f(C)$ at a point of $\Gamma$, there is necessarily a cubic surface $S$ containing $f(D)$ which is tangent to $f(C)$ at four points of $\Gamma$.

If $S$ were a multiple of $Q$, say $Q \cdot H$ where $H$ is a hyperplane, then since $f(C)$ is transverse to $Q$, it would follow that $H$ contains four points of $\Gamma$. But any 4 points on $f(C)$ are in linear general position. Consequently, $S$ is not a multiple of $Q$. Or equivalently, $f(D)=Q \cap S$ gives a presentation of $f(D)$ as a complete intersection.

If $S$ were tangent to $f(C)$ at all five points of $\Gamma$, then restricting the equation of $S$ to $f(C)$ would give a section of $\mathcal{O}_{C}(3) \simeq \mathcal{O}_{\mathbb{P}^{1}}(9)$ which vanished with multiplicity two at five points. Since the only such section is the zero section, we would conclude that $f(C) \subset S$. But then $f(C)$ would meet $f(D)$ at all 6 points of $f(C) \cap Q$, which we already ruled out above. Thus, $S$ is tangent to $f(C)$ at precisely four points of $\Gamma$.

Write $\Delta$ for the divisor on $D$ defined by these four points, and $p$ for the fifth point. Note that for $q \neq p$ in the tangent line to $\left(f_{1}, \Delta \cup\{p\}\right)$ at $p$,

$$
\begin{aligned}
\left.N_{f}\right|_{D} & \simeq\left(N_{f(D) / S}(\Delta+p) \oplus N_{f(D) / Q}(p)\right)[p \rightarrow q] \\
& \simeq\left(\mathcal{O}_{D}(2)(\Delta+p) \oplus \mathcal{O}_{D}(3)(p)\right)[p \rightarrow q] \\
\left.\Rightarrow N_{f}\right|_{D}(-2) & \simeq\left(\mathcal{O}_{D}(\Delta+p) \oplus \mathcal{O}_{D}(1)(p)\right)[p \rightarrow q] \\
& \simeq\left(\mathcal{O}_{D}(\Delta+p) \oplus K_{D}(p)\right)[p \rightarrow q] .
\end{aligned}
$$

By Riemann-Roch, $\operatorname{dim} H^{0}\left(K_{D}(p)\right)=4=\operatorname{dim} H^{0}\left(K_{D}\right)$; so every section of $K_{D}(p)$ vanishes at $p$. Consequently, the fiber of every section of $\mathcal{O}_{D}(\Delta+p) \oplus K_{D}(p)$ at $p$ lies in the fiber of the first factor. Since the fiber $\left.N_{f_{2} \rightarrow q}\right|_{p}$ does not lie in the fiber of the first factor, we have an isomorphism

$$
H^{0}\left(\left.N_{f}\right|_{D}(-2)\right) \simeq H^{0}\left(\left(\mathcal{O}_{D}(\Delta+p) \oplus K_{D}(p)\right)(-p)\right) \simeq H^{0}\left(\mathcal{O}_{D}(\Delta)\right) \oplus H^{0}\left(K_{D}\right)
$$

Consequently,

$$
\operatorname{dim} H^{0}\left(\left.N_{f}\right|_{D}(-2)\right)=\operatorname{dim} H^{0}\left(\mathcal{O}_{D}(\Delta)\right)+\operatorname{dim} H^{0}\left(K_{D}\right)=1+4=5=\chi\left(\left.N_{f}\right|_{D}(-2)\right),
$$

which implies

$$
H^{1}\left(\left.N_{f}\right|_{D}(-2)\right)=0 .
$$

Next, we prove the surjectivity of the evaluation map

$$
\mathrm{ev}: H^{0}\left(N_{f_{2}}(-2)\right) \rightarrow \bigoplus_{x \in \Gamma}\left(\frac{T_{x}\left(\mathbb{P}^{r}\right)}{f_{*}\left(T_{x}\left(C \cup_{\Gamma} D\right)\right)}\right)
$$

For this, we use the isomorphism

$$
N_{f_{2}}(-2) \simeq N_{f(D) / \mathbb{P}^{3}}(-2) \simeq N_{f(D) / S}(-2) \oplus N_{f(D) / Q}(-2) \simeq \mathcal{O}_{D} \oplus K_{D}
$$

The restriction of ev to $H^{0}\left(N_{f(D) / S}(-2) \simeq \mathcal{O}_{D}\right)$ maps trivially into the quotient $\frac{T_{x}\left(\mathbb{P}^{r}\right)}{f_{*}\left(T_{x}\left(C \cup_{\Gamma} D\right)\right)}$ for $x \in \Delta$, since $S$ is tangent to $f(C)$ along $\Delta$. Because $S$ is not tangent to $f(C)$ at $p$, the restriction 
of ev to $H^{0}\left(N_{f(D) / S}(-2) \simeq \mathcal{O}_{D}\right)$ thus maps isomorphically onto the factor $\frac{T_{p}\left(\mathbb{P}^{r}\right)}{f_{*}\left(T_{p}\left(C \cup_{\Gamma} D\right)\right)}$. It is therefore sufficient to show that the evaluation map

$$
H^{0}\left(N_{f(D) / Q}(-2) \simeq K_{D}\right) \rightarrow \bigoplus_{x \in \Delta}\left(\frac{T_{x}\left(\mathbb{P}^{r}\right)}{f_{*}\left(T_{x}\left(C \cup_{\Gamma} D\right)\right)}\right)
$$

is surjective. Or equivalently, since $Q$ is not tangent to $f(C)$ at any $x \in \Delta$, that the evaluation map

$$
\left.H^{0}\left(K_{D}\right) \rightarrow K_{D}\right|_{\Delta}
$$

is surjective. But this is clear since $\operatorname{dim} H^{0}\left(K_{D}\right)=4=\# \Delta$ and $\Delta$ is a general effective divisor of degree 4 on $D$.

Lemma 5.5. Let $f: C \rightarrow \mathbb{P}^{4}$ be a general $B N$-curve in $\mathbb{P}^{4}$, of arbitrary degree and genus. Then we can specialize $f$ to an unramified map from a reducible curve $f^{\circ}: C^{\prime} \cup L_{1} \cup L_{2} \cup L_{3} \rightarrow \mathbb{P}^{4}$, so that each $L_{i}$ is rational, $\left.f^{\circ}\right|_{L_{i}}$ is of degree 1 , and the images of the $L_{i}$ under $f^{\circ}$ are in linear general position.

Proof. By Lemma 4.8, our problem reduces to the case $f: C \rightarrow \mathbb{P}^{4}$ is a rational normal curve.

In this case, we begin by taking three general lines in $\mathbb{P}^{4}$. The locus of lines meeting each of our lines has class $\sigma_{2}$ in the Chow ring of the Grassmannian $\mathbb{G}(1,4)$ of lines in $\mathbb{P}^{4}$. By the standard calculus of Schubert cycles, we have $\sigma_{2}^{3}=\sigma_{2,2} \neq 0$ in the Chow ring of $\mathbb{G}(1,4)$. Thus, there exists a line meeting each of our three given lines. The (immersion of the) union of these four lines is then a specialization of a rational normal curve.

Lemma 5.6. Let $\Gamma \subset \mathbb{P}^{4}$ be a set of 6 points in linear general position; $\left(f_{1}: C \rightarrow \mathbb{P}^{4}, \Gamma_{1}\right)$ be either a general marked immersion of three disjoint lines, or a general marked BN-curve in $\mathbb{P}^{4}$, passing through $\Gamma$; and $\left(f_{2}: D \rightarrow \mathbb{P}^{4}, \Gamma_{2}\right)$ be a general marked canonical curve passing through $\Gamma$. If $H^{1}\left(N_{f_{1}}(-1)\right)=0$, then $f: C \cup_{\Gamma} D \rightarrow \mathbb{P}^{4}$ satisfies $H^{1}\left(N_{f}(-1)\right)=0$.

Proof. By Lemma 2.6, it suffices to prove that the natural map

$$
H^{0}\left(N_{f_{2}}(-1)\right) \rightarrow \bigoplus_{p \in \Gamma}\left(\frac{T_{p}\left(\mathbb{P}^{r}\right)}{f_{*}\left(T_{p}\left(C \cup_{\Gamma} D\right)\right)}\right)
$$

is surjective, and that

$$
H^{1}\left(\left.N_{f}\right|_{D}(-1)\right)=0 .
$$

These conditions both being open, we may apply Lemma 5.5 to specialize $\left(f_{1}, \Gamma_{1}\right)$ to a marked curve with reducible source $\left(f_{1}^{\circ}: C_{1} \cup C_{2} \rightarrow \mathbb{P}^{r}, \Gamma_{1}^{\circ}\right)$, with $C_{1}=L_{1} \cup L_{2} \cup L_{3}$ a union of 3 disjoint lines, and $\Gamma_{1}^{\circ} \subset C_{1}$ with 2 points on each line. It thus suffices to prove the above statements in the case when $C=C_{1}=L_{1} \cup L_{2} \cup L_{3}$ is the union of 3 general lines. Write $\Gamma=\Gamma_{1} \cup \Gamma_{2} \cup \Gamma_{3}$, where $\Gamma_{i} \subset L_{i}$.

It is well known that every canonical curve in $\mathbb{P}^{4}$ is the complete intersection of three quadrics; write $V$ for the vector space of quadrics vanishing along $f(D)$. For any 2 -secant line $L$ to $f(D)$, it is evident that it is one linear condition on quadrics in $V$ to contain $L$; and moreover, that general lines impose independent conditions unless there is a quadric which 
contains all 2-secant lines. Now the projection from a general line in $\mathbb{P}^{4}$ of $f(D)$ yields a nodal plane curve of degree 8 and geometric genus 5 , which in particular must have

$$
\left(\begin{array}{c}
8-1 \\
2
\end{array}\right)-5=16
$$

nodes. Consequently, the secant variety to $f(D)$ is a hypersurface of degree 16; and is thus not contained in a quadric. Thus, vanishing on general lines impose independent conditions on $V$. As $f\left(L_{1}\right), f\left(L_{2}\right)$, and $f\left(L_{3}\right)$ are general, we may thus choose a basis $V=\left\langle Q_{1}, Q_{2}, Q_{3}\right\rangle$ so that $Q_{i}$ contains $L_{j}$ if an only if $i \neq j$ (where the $Q_{i}$ are uniquely defined up to scaling). By construction, $f(D)$ is the complete intersection $Q_{1} \cap Q_{2} \cap Q_{3}$.

We now consider the direct sum decomposition

$$
N_{f_{2}} \simeq N_{f(D) / \mathbb{P}^{4}} \simeq N_{f(D) /\left(Q_{1} \cap Q_{2}\right)} \oplus N_{f(D) /\left(Q_{2} \cap Q_{3}\right)} \oplus N_{f(D) /\left(Q_{3} \cap Q_{1}\right)},
$$

which induces a direct sum decomposition

$$
\left.N_{f}\right|_{D} \simeq N_{f(D) /\left(Q_{1} \cap Q_{2}\right)}\left(\Gamma_{3}\right) \oplus N_{f(D) /\left(Q_{2} \cap Q_{3}\right)}\left(\Gamma_{1}\right) \oplus N_{f(D) /\left(Q_{3} \cap Q_{1}\right)}\left(\Gamma_{2}\right) .
$$

To show that $H^{1}\left(\left.N_{f}\right|_{D}(-1)\right)=0$, it is sufficient by symmetry to show that

$$
H^{1}\left(N_{f(D) /\left(Q_{1} \cap Q_{2}\right)}\left(\Gamma_{3}\right)(-1)\right)=0 .
$$

But we have

$$
N_{f(D) /\left(Q_{1} \cap Q_{2}\right)}\left(\Gamma_{3}\right)(-1) \simeq \mathcal{O}_{D}(2)\left(\Gamma_{3}\right)(-1) \simeq \mathcal{O}_{D}(1)\left(\Gamma_{3}\right)=K_{D}\left(\Gamma_{3}\right)
$$

so by Serre duality,

$$
H^{1}\left(N_{f(D) /\left(Q_{1} \cap Q_{2}\right)}\left(\Gamma_{3}\right)(-1)\right) \simeq H^{0}\left(\mathcal{O}_{D}\left(-\Gamma_{3}\right)\right)^{\vee}=0 .
$$

Next, we examine the evaluation map

$$
H^{0}\left(N_{f_{2}}(-1)\right) \rightarrow \bigoplus_{p \in \Gamma}\left(\frac{T_{p}\left(\mathbb{P}^{r}\right)}{f_{*}\left(T_{p}\left(C \cup_{\Gamma} D\right)\right)}\right)
$$

For this, we use the direct sum decomposition

$$
N_{f_{2}} \simeq N_{f(D) / \mathbb{P}^{4}} \simeq N_{f(D) /\left(Q_{1} \cap Q_{2}\right)}(-1) \oplus N_{f(D) /\left(Q_{2} \cap Q_{3}\right)}(-1) \oplus N_{f(D) /\left(Q_{3} \cap Q_{1}\right)}(-1),
$$

together with the decomposition (for $p \in \Gamma_{i}$ ):

$$
\left.\frac{T_{p}\left(\mathbb{P}^{r}\right)}{f_{*}\left(T_{p}\left(C \cup_{\Gamma_{i}} L_{i}\right)\right)} \simeq \bigoplus_{j \neq i} N_{f(D) /\left(Q_{i} \cap Q_{j}\right)}\right|_{p}
$$

This reduces our problem to showing (by symmetry) the surjectivity of

$$
\left.H^{0}\left(N_{f(D) /\left(Q_{1} \cap Q_{2}\right)}(-1)\right) \rightarrow \bigoplus_{p \in \Gamma_{1} \cup \Gamma_{2}} N_{f(D) /\left(Q_{1} \cap Q_{2}\right)}\right|_{p}
$$


But for this, it is sufficient to note that $\Gamma_{1} \cup \Gamma_{2}$ is a general collection of 4 points on $D$, and

$$
N_{f(D) /\left(Q_{1} \cap Q_{2}\right)}(-1) \simeq \mathcal{O}_{D}(2)(-1)=\mathcal{O}_{D}(1) \simeq K_{D}
$$

It thus remains to show

$$
\left.H^{0}\left(K_{D}\right) \rightarrow K_{D}\right|_{\Gamma_{1} \cup \Gamma_{2}}
$$

is surjective, where $\Gamma_{1} \cup \Gamma_{2}$ is a general collection of 4 points on $D$. But this is clear because $K_{D}$ is a line bundle and $\operatorname{dim} H^{0}\left(K_{D}\right)=5 \geq 4$.

Corollary 5.7. To prove the main theorems (excluding the "conversely..." part), it suffices to verify them in the following special cases:

1. For Theorem 1.4, it suffices to consider the cases where $(d, g)$ is one of:

$$
\begin{aligned}
& (5,1), \quad(7,2), \quad(6,3), \quad(7,4), \quad(8,5), \quad(9,6), \quad(9,7), \\
& (10,9), \quad(11,10), \quad(12,12), \quad(13,13) \quad(14,14) \text {. }
\end{aligned}
$$

2. For Theorem 1.5, it suffices to consider the cases where $(d, g)$ is one of:

$$
(7,5), \quad(8,6)
$$

3. For Theorem [1.6, it suffices to consider the cases where $(d, g)$ is one of:

$$
(9,5), \quad(10,6), \quad(11,7), \quad(12,9), \quad(16,15), \quad(17,16), \quad(18,17) .
$$

In proving the theorems in each of these cases, we may suppose the corresponding theorem holds for curves of smaller genus.

Proof. For Theorem 1.4, note that by Lemma 5.2 and Proposition 4.2, it suffices to show Theorem 1.4 for each pair $(d, g)$, where $d$ is minimal (i.e., where $\rho(d, g)=\rho(d, g, r=3) \geq 0$ and $(d, g)$ is not in our list of counterexamples; but either $\rho(d-1, g)<0$, or $(d-1, g)$ is in our list of counterexamples).

If $\rho(d, g) \geq 0$ and $g \geq 15$, then $(d-6, g-8)$ is not in our list of counterexamples, and $\rho(d-6, g-8)=\rho(d, g) \geq 0$. By induction, we know $H^{1}\left(N_{f}(-2)\right)=0$ for $f$ a BN-general curve of degree $d-6$ and genus $g-8$. Applying Lemma 5.3 (and Proposition 4.2), we conclude the desired result. If $\rho(d, g) \geq 0$ and $g \leq 14$, and $d$ is minimal as above, then either $(d, g)$ is in our above list, or $(d, g) \in\{(3,0),(9,8),(12,11)\}$. The case of $(d, g)=(3,0)$ follows from Lemma 3.5. But in these last two cases, Lemma 5.3 again implies the desired result (using Theorem 1.4 for $\left(d^{\prime}, g^{\prime}\right)=(d-6, g-8)$ as our inductive hypotheses).

For Theorem 1.5, we note that if $H^{1}\left(N_{f}(-2)\right)=0$, then it follows that $H^{1}\left(N_{f}(-1)\right)=0$. It therefore suffices to check the list of counterexamples appearing in Theorem 1.4 besides the counterexample $(d, g)=(6,4)$ listed in Theorem 1.5. The cases $(d, g) \in\{(4,1),(5,2),(6,2)\}$ follow from Lemma 3.5, so we only have to consider the remaining cases (which form the given list).

Finally, for Theorem 1.6, Lemma 5.1 implies it suffices to show Theorem 1.6 for each pair $(d, g)$ with $d$ minimal. If $\rho(d, g) \geq 0$ and $g \geq 18$, then $(d-8, g-10)$ is not in our list of 
counterexamples, and $\rho(d-8, g-10)=\rho(d, g) \geq 0$. By induction, we know $H^{1}\left(N_{f}(-1)\right)=0$ for $C$ is a general curve of degree $d-8$ and genus $g-10$. Applying Lemma 5.6, we conclude the desired result. If $\rho(d, g) \geq 0$ and $g \leq 17$, and $d$ is minimal as above, then either $(d, g)$ is in our above list, or

$$
(d, g) \in\{(4,0),(5,1),(6,2),(7,3),(8,4)\}
$$

or

$$
(d, g) \in\{(11,8),(12,10),(13,11),(14,12),(15,13),(16,14)\},
$$

In the first set of cases above, Lemma 3.5 implies the desired result. But in the last set of cases, Lemma 5.6 again implies the desired result. Here, for $(d, g)=(11,8)$, our inductive hypothesis is that $H^{1}\left(N_{f}(-1)\right)=0$ for $f: L_{1} \cup L_{2} \cup L_{3} \rightarrow \mathbb{P}^{4}$ an immersion of three skew lines. In the remaining cases, we use Theorem 1.4 for $\left(d^{\prime}, g^{\prime}\right)=(d-8, g-10)$ as our inductive hypothesis.

\section{Adding Curves in a Hyperplane}

In this section, we explain an inductive strategy involving adding curves contained in hyperplanes, which will help resolve many of our remaining cases.

Lemma 6.1. Let $H \subset \mathbb{P}^{r}$ (for $r \geq 3$ ) be a hyperplane, and let $\left(f_{1}: C \rightarrow \mathbb{P}^{r}, \Gamma_{1}\right)$ and $\left(f_{2}: D \rightarrow H, \Gamma_{2}\right)$ be marked curves, both passing through a set $\Gamma \subset H \subset \mathbb{P}^{r}$ of $n \geq 1$ points.

Assume that $f_{2}$ is a general BN-curve of degree $d$ and genus $g$ to $H$, that $\Gamma_{2}$ is a general collection of $n$ points on $D$, and that $f_{1}$ is transverse to $H$ along $\Gamma$. If

$$
H^{1}\left(N_{f_{1}}(-\Gamma)\right)=0 \quad \text { and } \quad n \geq g-d+r,
$$

then $f: C \cup_{\Gamma} D \rightarrow \mathbb{P}^{r}$ satisfies $H^{1}\left(N_{f}\right)=0$ and is a limit of unramified maps from smooth curves.

If in addition $f_{1}$ is an immersion, $f(C) \cap f(D)$ is exactly equal to $\Gamma$, and $\mathcal{O}_{D}(1)(\Gamma)$ is very ample away from $\Gamma$ - i.e. if $\operatorname{dim} H^{0}\left(\mathcal{O}_{D}(1)(\Gamma)(-\Delta)\right)=\operatorname{dim} H^{0}\left(\mathcal{O}_{D}(1)(\Gamma)\right)-2$ for any effective divisor $\Delta$ of degree 2 supported on $D \backslash \Gamma$ - then $f$ is a limit of immersions of smooth curves.

Remark 6.2. The condition that $\mathcal{O}_{D}(1)(\Gamma)$ is very ample away from $\Gamma$ is immediate when $\mathcal{O}_{D}(1)$ is very ample (which in particular happens for $r \geq 4$ ). It is also immediate when $n \geq g$, in which case $\mathcal{O}_{D}(1)(\Gamma)$ is a general line bundle of degree $d+n \geq g+r \geq g+3$ and is thus very ample.

Proof. Note that $N_{f_{1}}$ is a subsheaf of $\left.N_{f}\right|_{C}$ with punctual quotient (supported at $\Gamma$ ). Twisting down by $\Gamma$, we obtain a short exact sequence

$$
\left.0 \rightarrow N_{f_{1}}(-\Gamma) \rightarrow N_{f}\right|_{C}(-\Gamma) \rightarrow * \rightarrow 0,
$$

where $*$ denotes a punctual sheaf, which in particular has vanishing $H^{1}$. Since $H^{1}\left(N_{f_{1}}(-\Gamma)\right)=0$ by assumption, we conclude that $H^{1}\left(\left.N_{f}\right|_{C}(-\Gamma)\right)=0$ too. Since $f_{2}$ is a general BN-curve, $H^{1}\left(N_{f_{2}}\right)=0$. The exact sequences

$$
\begin{gathered}
\left.\left.0 \rightarrow N_{f}\right|_{C}(-\Gamma) \rightarrow N_{f} \rightarrow N_{f}\right|_{D} \rightarrow 0 \\
\left.\left.0 \rightarrow N_{f_{2}} \rightarrow N_{f}\right|_{D} \rightarrow N_{H}\right|_{D}(\Gamma) \simeq \mathcal{O}_{D}(1)(\Gamma) \rightarrow 0
\end{gathered}
$$


then imply that, to check $H^{1}\left(N_{f}\right)=0$, it suffices to check $H^{1}\left(\mathcal{O}_{D}(1)(\Gamma)\right)=0$. They moreover imply that every section of $\left.N_{H}\right|_{D}(\Gamma) \simeq \mathcal{O}_{D}(1)(\Gamma)$ lifts to a section of $N_{f}$, which, as $H^{1}\left(N_{f}\right)=0$, lifts to a global deformation of $f$.

To check $f$ is a limit of unramified maps from smooth curves, it remains to see that the generic section of $\left.N_{H}\right|_{D}(\Gamma) \simeq \mathcal{O}_{D}(1)(\Gamma)$ corresponds to a first-order deformation which smoothes the nodes $\Gamma$ - or equivalently does not vanish at $\Gamma$.

Since by assumption $f_{1}$ is an immersion and there are no other nodes where $f(C)$ and $f(D)$ meet besides $\Gamma$, to see that $f$ is a limit of immersions of smooth curves, it remains to note in addition that the generic section of $\left.N_{H}\right|_{D}(\Gamma) \simeq \mathcal{O}_{D}(1)(\Gamma)$ separates the points of $D$ identified under $f_{2}$ - which is true by assumption that $\mathcal{O}_{D}(1)(\Gamma)$ is very ample away from $\Gamma$.

To finish the proof, it thus suffices to check $H^{1}\left(\mathcal{O}_{D}(1)(\Gamma)\right)=0$, and that the generic section of $\mathcal{O}_{D}(1)(\Gamma)$ does not vanish at any point $p \in \Gamma$. Equivalently, it suffices to check $H^{1}\left(\mathcal{O}_{D}(1)(\Gamma)(-p)\right)=0$ for $p \in \Gamma$. Since $f_{2}$ is a general BN-curve, we obtain

$$
\operatorname{dim} H^{1}\left(\mathcal{O}_{D}(1)\right)=\max (0, g-d+(r-1)) \leq n-1 .
$$

Twisting by $\Gamma \backslash\{p\}$, which is a set of $n-1$ general points, we therefore obtain

$$
H^{1}\left(\mathcal{O}_{D}(1)(\Gamma \backslash\{p\})\right)=0,
$$

as desired.

Lemma 6.3. Let $k \geq 1$ be an integer, $\iota: H \hookrightarrow \mathbb{P}^{r}(r \geq 3)$ be a hyperplane, and $\left(f_{1}: C \rightarrow \mathbb{P}^{r}, \Gamma_{1}\right)$ and $\left(f_{2}: D \rightarrow H, \Gamma_{2}\right)$ be marked curves, both passing through a set $\Gamma \subset H \subset \mathbb{P}^{r}$ of $n \geq 1$ points.

Assume that $f_{2}$ is a general BN-curve of degree $d$ and genus $g$ to $H$, that $\Gamma_{2}$ is a general collection of $n$ points on $D$, and that $f_{1}$ is transverse to $H$ along $\Gamma$. Suppose moreover that:

1. The bundle $N_{f_{2}}(-k)$ satisfies interpolation.

2. We have $H^{1}\left(N_{f_{1}}(-k)\right)=0$.

3. We have

$$
(r-2) n \leq r d-(r-4)(g-1)-k \cdot(r-2) d .
$$

4. We have

$$
n \geq \begin{cases}g & \text { if } k=1 \\ g-1+(k-1) d & \text { if } k>1 .\end{cases}
$$

Then $f: C \cup_{\Gamma} D \rightarrow \mathbb{P}^{r}$ satisfies

$$
H^{1}\left(N_{f}(-k)\right)=0 .
$$

Proof. Since $N_{f_{2}}(-k)$ satisfies interpolation by assumption and

$$
(r-2) n \leq \chi\left(N_{f_{2}}(-k)\right)=r d-(r-4)(g-1)-k \cdot(r-2) d,
$$

we conclude that $H^{1}\left(N_{f_{2}}(-k)(-\Gamma)\right)=0$. Since $H^{1}\left(N_{f_{1}}(-k)\right)=0$ by assumption, to apply Lemma 2.7 it remains to check

$$
H^{1}\left(\mathcal{O}_{D}(1-k)(\Gamma)\right)=0 .
$$


It is therefore sufficient for

$$
n=\# \Gamma \geq \operatorname{dim} H^{1}\left(\mathcal{O}_{D}(1-k)\right)= \begin{cases}g & \text { if } k=1 \\ g-1+(k-1) d & \text { if } k>1\end{cases}
$$

But this is precisely our final assumption.

\section{Curves of Large Genus}

In this section, we will deal with a number of our special cases, of larger genus. Taking care of these cases separately is helpful - since in the remaining cases, we will not have to worry about whether our curve is a BN-curve, thanks to results of [7] and [8] on the irreducibility of the Hilbert scheme of curves.

Lemma 7.1. Let $H \subset \mathbb{P}^{3}$ be a plane, $\Gamma \subset H \subset \mathbb{P}^{3}$ a set of 6 general points, $\left(f_{1}: C \rightarrow \mathbb{P}^{3}, \Gamma_{1}\right)$ a general marked BN-curve passing through $\Gamma$ of degree and genus one of

$$
(d, g) \in\{(6,1),(7,2),(8,4),(9,5),(10,6)\},
$$

and $\left(f_{2}: D \rightarrow H, \Gamma_{2}\right)$ a general marked canonical curve passing through $\Gamma$. Then $f: C \cup_{\Gamma} D \rightarrow \mathbb{P}^{3}$ is a $B N$-curve which satisfies $H^{1}\left(N_{f}\right)=0$.

Proof. Note that the conclusion is an open condition; we may therefore freely specialize $\left(f_{1}, \Gamma_{1}\right)$. Write $\Gamma=\{s, t, u, v, w, x\}$.

In the case $(d, g)=(6,1)$, we specialize $\left(f_{1}, \Gamma_{1}\right)$ to $\left(f_{1}^{\circ}: C^{\circ}=C_{1} \cup_{p} C_{2} \cup_{\{q, r\}} C_{3} \rightarrow \mathbb{P}^{3}, \Gamma_{1}^{\circ}\right)$, where $\left.f_{1}^{\circ}\right|_{C_{1}}$ is a conic, $\left.f_{1}^{\circ}\right|_{C_{2}}$ is a line with $C_{2}$ joined to $C_{1}$ at one point $p$, and $\left.f_{1}^{\circ}\right|_{C_{3}}$ is a rational normal curve with $C_{3}$ joined to $C_{1}$ at two points $\{q, r\}$; note that $f_{1}^{\circ}$ is a BN-curve by (iterative application of) Proposition 4.2. We suppose that $\left(\left.f_{1}^{\circ}\right|_{C_{1}}, \Gamma_{1}^{\circ} \cap C_{1}\right)$ passes through $\{s, t\}$, while $\left(\left.f_{1}^{\circ}\right|_{C_{2}}, \Gamma_{1}^{\circ} \cap C_{2}\right)$ passes through $u$, and $\left(\left.f_{1}^{\circ}\right|_{C_{3}}, \Gamma_{1}^{\circ} \cap C_{3}\right)$ passes through $\{v, w, x\}$; it is clear this can be done so $\{s, t, u, v, w, x\}$ are general. Writing

$$
f^{\circ}: C^{\circ} \cup_{\Gamma} D=C_{2} \cup_{\{p, u\}} C_{3} \cup_{\{q, r, v, w, x\}}\left(C_{1} \cup_{\{s, t\}} D\right) \rightarrow \mathbb{P}^{3},
$$

it suffices by Propositions 4.2 and 4.3 to show that $\left.f^{\circ}\right|_{C_{1} \cup D}$ is a BN-curve which satisfies $H^{1}\left(N_{\left.f^{\circ}\right|_{C_{1} \cup D}}\right)=0$.

For $(d, g)=(8,4)$, we specialize $\left(f_{1}, \Gamma_{1}\right)$ to $\left(f_{1}^{\circ}: C^{\circ}=C_{1} \cup_{\{p, q, r\}} C_{2} \cup_{\{y, z, a\}} C_{3} \rightarrow \mathbb{P}^{3}, \Gamma_{1}^{\circ}\right)$, where $\left.f_{1}^{\circ}\right|_{C_{1}}$ is a conic, and $\left.f_{1}^{\circ}\right|_{C_{2}}$ and $\left.f_{1}^{\circ}\right|_{C_{3}}$ are rational normal curves, with both $C_{2}$ and $C_{3}$ joined to $C_{1}$ at 3 points (at $\{p, q, r\}$ and $\{y, z, a\}$ respectively); note that $f_{1}^{\circ}$ is a BN-curve by (iterative application of) Proposition 4.2. We suppose that $\left(\left.f_{1}^{\circ}\right|_{C_{1}}, \Gamma_{1}^{\circ} \cap C_{1}\right)$ passes through $\{s, t\}$, while $\left(\left.f_{1}^{\circ}\right|_{C_{2}}, \Gamma_{1}^{\circ} \cap C_{2}\right)$ passes through $\{u, v\}$, and $\left(\left.f_{1}^{\circ}\right|_{C_{3}}, \Gamma_{1}^{\circ} \cap C_{3}\right)$ passes through $\{w, x\}$; it is clear this can be done so $\{s, t, u, v, w, x\}$ are general. Writing

$$
f^{\circ}: C^{\circ} \cup_{\Gamma} D=C_{2} \cup_{\{p, q, r, u, v\}} C_{3} \cup_{\{w, x, y, z, a\}}\left(C_{1} \cup_{\{s, t\}} D\right) \rightarrow \mathbb{P}^{3},
$$

it again suffices by Propositions 4.2 and 4.3 to show that $\left.f^{\circ}\right|_{C_{1} \cup D}$ is a BN-curve which satisfies $H^{1}\left(N_{\left.f^{\circ}\right|_{C_{1} \cup D}}\right)=0$. 
For this, we first note that $\left.f^{\circ}\right|_{C_{1} \cup D}$ is a curve of degree 6 and genus 4 , and that the moduli space of smooth curves of degree 6 and genus 4 in $\mathbb{P}^{3}$ is irreducible (they are all canonical curves). Moreover, by Lemma 6.1 (c.f. Remark 6.2 and note that $\mathcal{O}_{D}(1) \simeq K_{D}$ is very ample), $\left.f^{\circ}\right|_{C_{1} \cup D}$ is a limit of immersions of smooth curves, and satisfies $H^{1}\left(N_{\left.f^{\circ}\right|_{C_{1} \cup D}}\right)=0$; this completes the proof.

Lemma 7.2. Let $H \subset \mathbb{P}^{4}$ be a hyperplane, $\Gamma \subset H \subset \mathbb{P}^{4}$ a set of 7 general points, $\left(f_{1}: C \rightarrow \mathbb{P}^{4}, \Gamma_{1}\right)$ a general marked BN-curve passing through $\Gamma$ of degree and genus one of

$$
(d, g) \in\{(7,3),(8,4),(9,5)\}
$$

and $\left(f_{2}: D \rightarrow H, \Gamma_{2}\right)$ a general marked BN-curve of degree 9 and genus 6 passing through $\Gamma$. Then $f: C \cup_{\Gamma} D \rightarrow \mathbb{P}^{4}$ is a BN-curve which satisfies $H^{1}\left(N_{f}\right)=0$.

Proof. Again, we note that the conclusion is an open statement; we may therefore freely specialize $\left(f_{1}, \Gamma_{1}\right)$. Write $\Gamma=\{t, u, v, w, x, y, z\}$.

First, we claim it suffices to consider the case $(d, g)=(7,3)$. Indeed, suppose $\left(f_{1}, \Gamma_{1}\right)$ is a marked BN-curve of degree 7 and genus 3 passing through $\Gamma$. Then $f_{1}^{\prime}: C \cup_{\{p, q\}} L \rightarrow \mathbb{P}^{4}$ and $f_{1}^{\prime \prime}: C \cup_{\{p, q\}} L \cup_{\{r, s\}} L^{\prime} \rightarrow \mathbb{P}^{4}$ (where $\left.f_{1}^{\prime}\right|_{L}$ and $\left.f_{1}^{\prime \prime}\right|_{L}$ and $\left.f_{1}^{\prime \prime}\right|_{L^{\prime}}$ are lines with $L$ and $L^{\prime}$ joined to $C$ at two points) are BN-curves by Proposition 4.2, of degree and genus $(8,4)$ and $(9,5)$ respectively. If $f: C \cup_{\Gamma} D \rightarrow \mathbb{P}^{4}$ is a BN-curve with $H^{1}\left(N_{f}\right)=0$, then invoking Propositions 4.2 and 4.3 , both

$$
f^{\prime}:\left(C \cup_{\{p, q\}} L\right) \cup_{\Gamma} D=\left(C \cup_{\Gamma} D\right) \cup_{\{p, q\}} L \rightarrow \mathbb{P}^{4}
$$

and $f^{\prime \prime}:\left(C \cup_{\{p, q\}} L \cup_{\{r, s\}} L^{\prime}\right) \cup_{\Gamma} D=\left(C \cup_{\Gamma} D\right) \cup_{\{p, q\}} L \cup_{\{r, s\}} L^{\prime} \rightarrow \mathbb{P}^{4}$

are BN-curves, which satisfy $H^{1}\left(N_{f^{\prime}}\right)=H^{1}\left(N_{f^{\prime \prime}}\right)=0$.

So it remains to consider the case $(d, g)=(7,3)$. In this case, we begin by specializing $\left(f_{1}, \Gamma_{1}\right)$ to $\left(f_{1}^{\circ}: C^{\circ}=C^{\prime} \cup_{\{p, q\}} L \rightarrow \mathbb{P}^{4}, \Gamma_{1}^{\circ}\right)$, where $\left.f_{1}^{\circ}\right|_{C^{\prime}}$ is a general BN-curve of degree 6 and genus 2 , and $\left.f_{1}^{\circ}\right|_{L}$ is a line with $L$ joined to $C^{\prime}$ at two points $\{p, q\}$. We suppose that $\left(\left.f_{1}^{\circ}\right|_{L}, \Gamma_{1}^{\circ} \cap L\right)$ passes through $t$, while $\left(\left.f_{1}^{\circ}\right|_{C^{\prime}}, \Gamma_{1}^{\circ} \cap C^{\prime}\right)$ passes through $\{u, v, w, x, y, z\}$; we must check this can be done so $\{t, u, v, w, x, y, z\}$ are general. To see this, it suffices to show that the intersection $f_{1}^{\circ}\left(C^{\prime}\right) \cap H$ and the points $\left\{f_{1}^{\circ}(p), f_{1}^{\circ}(q)\right\}$ independently general. In other words, we are claiming that the map

$$
\left\{\left(\left.f_{1}^{\circ}\right|_{C^{\prime}}: C^{\prime} \rightarrow \mathbb{P}^{4}, p, q\right): p, q \in C^{\prime}\right\} \mapsto\left(\left.f_{1}^{\circ}\right|_{C^{\prime}}\left(C^{\prime}\right) \cap H,\left.f_{1}^{\circ}\right|_{C^{\prime}}(p),\left.f_{1}^{\circ}\right|_{C^{\prime}}(q)\right)
$$

is dominant; equivalently, that it is smooth at a generic point $\left(\left.f_{1}^{\circ}\right|_{C^{\prime}}, p, q\right)$. But the obstruction to smoothness lies in $H^{1}\left(N_{\left.f_{1}^{\circ}\right|_{C^{\prime}}}(-1)(-p-q)\right)=0$, which vanishes because because $N_{\left.f_{1}^{\circ}\right|_{C^{\prime}}}(-1)$ satisfies interpolation by Lemma 3.5 .

We next specialize $\left(f_{2}, \Gamma_{2}\right)$ to $\left(f_{2}^{\circ}: D^{\circ}=D^{\prime} \cup_{\Delta} D_{1} \rightarrow H, \Gamma_{2}^{\circ}\right)$, where $\left.f_{2}^{\circ}\right|_{D^{\prime}}$ is a general BNcurve of degree 6 and genus 3 , and $\left.f_{2}^{\circ}\right|_{D_{1}}$ is a rational normal curve with $D_{1}$ joined to $D^{\prime}$ at a set $\Delta$ of 4 points; note that $f_{2}^{\circ}$ is a BN-curve by Proposition 4.2. We suppose that $\left(\left.f_{2}^{\circ}\right|_{D_{1}}, \Gamma_{2}^{\circ} \cap D_{1}\right)$ passes through $t$, while $\left(\left.f_{2}^{\circ}\right|_{D^{\prime}}, \Gamma_{2}^{\circ} \cap D^{\prime}\right)$ passes through $\{u, v, w, x, y, z\}$; this can be done so $\{t, u, v, w, x, y, z\}$ are still general, since $\left.f_{2}^{\circ}\right|_{D^{\prime}}$ (marked at general points of the source) can pass through 6 general points, while $\left(\left.f_{2}^{\circ}\right|_{D_{1}}\right.$ (again marked at general points of the source) can pass through 5 general points, both by Corollary 1.4 of [2]. 
In addition, $\left(\left.f_{2}^{\circ}\right|_{D_{1}},\left(\hat{t}=\Gamma_{2}^{\circ} \cap D_{1}\right) \cup \Delta\right)$ has a general tangent line at $t$; to see this, note that we are asserting that the map sending $\left(\left.f_{2}^{\circ}\right|_{D_{1}}, \hat{t} \cup \Delta\right)$ to its tangent line at $t$ is dominant; equivalently, that it is smooth at a generic point of the source. But the obstruction to smoothness lies in $H^{1}\left(N_{\left.f_{2}^{\circ}\right|_{D_{1}}}(-\Delta-2 \hat{t})\right)$, which vanishes because $N_{\left.f_{2}^{\circ}\right|_{D_{1}}}(-2 \hat{t})$ satisfies interpolation by combining Propositions 3.2 and 3.3 .

As $\{p, q\} \subset C^{\prime}$ is general, we thus know that the tangent lines to $\left(\left.f_{2}^{\circ}\right|_{D_{1}}, \hat{t} \cup \Delta\right)$ at $t$, and to $\left(\left.f_{1}^{\circ}\right|_{C^{\prime}},\{p, q\}\right)$ at $f_{1}^{\circ}(p)$ and $f_{1}^{\circ}(q)$, together span all of $\mathbb{P}^{4}$; write $\bar{t}, \bar{p}$, and $\bar{q}$ for points on each of these tangent lines distinct from $t, f_{1}^{\circ}(p)$, and $f_{1}^{\circ}(q)$ respectively. We then use the exact sequences

$$
\begin{aligned}
& \left.\left.0 \rightarrow N_{f \circ}\right|_{L}(-\hat{t}-p-q) \rightarrow N_{f^{\circ}} \rightarrow N_{f^{\circ}}\right|_{C^{\prime} \cup D^{\circ}} \rightarrow 0 \\
& \left.\left.0 \rightarrow N_{f^{\circ}}\right|_{C^{\prime} \cup D^{\circ}} \rightarrow N_{f^{\circ}}\right|_{C^{\prime} \cup D^{\circ}} \rightarrow * \rightarrow 0,
\end{aligned}
$$

where $*$ is a punctual sheaf (which in particular has vanishing $H^{1}$ ). Write $H_{t}$ for the hyperplane spanned by $f_{1}^{\circ}(L), \bar{p}$, and $\bar{q}$; and $H_{p}$ for the hyperplane spanned by $f_{1}^{\circ}(L), \bar{t}$, and $\bar{q}$; and $H_{q}$ for the hyperplane spanned by $f_{1}^{\circ}(L), \bar{t}$, and $\bar{p}$. Then $f_{1}^{\circ}(L)$ is the complete intersection $H_{t} \cap H_{p} \cap H_{q}$, and so we get a decomposition

$$
\left.N_{f^{\circ}}\right|_{L} \simeq N_{f_{1}^{\circ}(L) / H_{t}}(\hat{t}) \oplus N_{f_{1}^{\circ}(L) / H_{p}}(p) \oplus N_{f_{1}^{\circ}(L) / H_{q}}(q),
$$

which upon twisting becomes

$$
\left.N_{f^{\circ}}\right|_{L}(-\hat{t}-p-q) \simeq N_{f_{1}^{\circ}(L) / H_{t}}(-p-q) \oplus N_{f_{1}^{\circ}(L) / H_{p}}(-\hat{t}-q) \oplus N_{f_{1}^{\circ}(L) / H_{q}}(-\hat{t}-p) .
$$

Note that $N_{f_{1}^{\circ}(L) / H_{t}}(-p-q) \simeq \mathcal{O}_{L}(-1)$ has vanishing $H^{1}$, and similarly for the other factors; consequently, $H^{1}\left(\left.N_{f^{\circ}}\right|_{L}(-\hat{t}-p-q)\right)=0$. We conclude that $H^{1}\left(N_{f} \circ\right)=0$ provided that $H^{1}\left(N_{\left.f^{\circ}\right|_{C^{\prime} \cup D^{\circ}}}\right)=0$. Moreover, writing $C^{\prime} \cup_{\{u, v, w, x, y, z\}} D^{\circ}=D_{1} \cup_{\Delta}\left(D^{\prime} \cup_{\{u, v, w, x, y, z\}} C^{\prime}\right)$ and

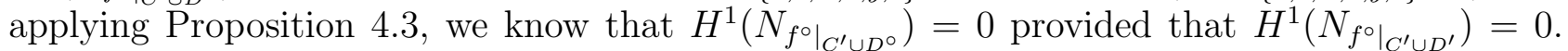
And if $\left.f^{\circ}\right|_{C^{\prime} \cup D^{\prime}}$ is a BN-curve, then $f^{\circ}:\left(C^{\prime} \cup_{\{u, v, w, x, y, z\}} D^{\prime}\right) \cup_{\Delta \cup\{p, q\}}\left(D_{1} \cup_{t} L\right) \rightarrow \mathbb{P}^{4}$ is a BNcurve too by Proposition 4.2, Putting this all together, it is sufficient to show that $\left.f^{\circ}\right|_{C^{\prime} \cup D^{\prime}}$ is a BN-curve which satisfies $H^{1}\left(\left.N_{f^{\circ}}\right|_{C^{\prime} \cup D^{\prime}}\right)=0$.

Our next step is to specialize $\left(\left.f_{1}^{\circ}\right|_{C^{\prime}}, \Gamma_{1}^{\circ} \cap C^{\prime}\right)$ to $\left(f_{1}^{\circ \circ}: C^{\circ \circ}=C^{\prime \prime} \cup_{\{r, s\}} L^{\prime} \rightarrow \mathbb{P}^{4}, \Gamma_{1}^{\circ \circ}\right)$, where $\left.f_{1}^{\circ \circ}\right|_{C^{\prime \prime}}$ is a general BN-curve of degree 5 and genus 1 , and $\left.f_{1}^{\circ \circ}\right|_{L^{\prime}}$ is a line with $L^{\prime}$ joined to $C^{\prime \prime}$ at two points $\{r, s\}$. We suppose that $\left(\left.f_{1}^{\circ \circ}\right|_{C^{\prime \prime}}, \Gamma_{1}^{\circ \circ} \cap C^{\prime \prime}\right)$ passes through $u$, while $\left(\left.f_{1}^{\circ \circ}\right|_{C^{\prime \prime}}, \Gamma_{1}^{\circ \circ} \cap C^{\prime \prime}\right)$ passes through $\{v, w, x, y, z\}$; as before this can be done so $\{u, v, w, x, y, z\}$ are general. We also specialize $\left(\left.f_{2}^{\circ}\right|_{D^{\prime}}, \Gamma_{2}^{\circ} \cap D^{\prime}\right)$ to $\left(f_{2}^{\circ \circ}: D^{\prime \prime} \cup_{\Delta} D_{2} \rightarrow \mathbb{P}^{4}, \Gamma_{2}^{\circ \circ}\right)$, where $\left.f_{2}^{\circ \circ}\right|_{D^{\prime \prime}}$ and $\left.f_{2}^{\circ \circ}\right|_{D_{2}}$ are both rational normal curves with $D^{\prime \prime}$ and $D_{2}$ joined at a set $\Delta$ of 4 general points. We suppose that $\left(\left.f_{2}^{\circ \circ}\right|_{D_{2}}, \Gamma_{2}^{\circ \circ} \cap D_{2}\right)$ passes through $u$, while $\left(\left.f_{2}^{\circ \circ}\right|_{D^{\prime \prime}}, \Gamma_{2}^{\circ \circ} \cap D^{\prime \prime}\right)$ passes through $\{v, w, x, y, z\}$; as before this can be done so $\{u, v, w, x, y, z\}$ are general. The same argument as above, mutatis mutandis, then implies it is sufficient to show that $\left.f^{\circ \circ}\right|_{C^{\prime \prime} \cup D^{\prime \prime}}: C^{\prime \prime} \cup_{\{v, w, x, y, z\}} D^{\prime \prime} \rightarrow \mathbb{P}^{4}$ is a BN-curve which satisfies $H^{1}\left(\left.N_{f \circ{ }^{\circ}}\right|_{C^{\prime \prime} \cup D^{\prime \prime}}\right)=0$.

For this, we first note that $\left.f^{\circ \circ}\right|_{C^{\prime \prime} \cup D^{\prime \prime}}$ is a curve of degree 8 and genus 5 , and that the moduli space of smooth curves of degree 8 and genus 5 in $\mathbb{P}^{4}$ is irreducible (they are all canonical curves). To finish the proof, it suffices to note by Lemma 6.1 that $\left.f^{\circ \circ}\right|_{C^{\prime \prime} \cup D^{\prime \prime}}$ is a limit of immersions of smooth curves and satisfies $H^{1}\left(N_{\left.f^{\circ \circ}\right|_{C^{\prime \prime} \cup D^{\prime \prime}}}\right)=0$. 
Corollary 7.3. To prove the main theorems (excluding the "conversely..." part), it suffices to show the existence of (nondegenerate immersions of) smooth curves, of the following degrees and genera, which satisfy the conclusions:

1. For Theorem 1.4, it suffices to show the existence of smooth curves, satisfying the conclusions, where $(d, g)$ is one of:

$$
(5,1), \quad(7,2), \quad(6,3), \quad(7,4), \quad(8,5), \quad(9,6), \quad(9,7) .
$$

2. For Theorem 1.5, it suffices to show the existence of smooth curves, satisfying the conclusions, where $(d, g)$ is one of:

$$
(7,5), \quad(8,6) .
$$

3. For Theorem 1.6, it suffices to show the existence of smooth curves, satisfying the conclusions, where $(d, g)$ is one of:

$$
(9,5), \quad(10,6), \quad(11,7), \quad(12,9) .
$$

(And in constructing the above smooth curves, we may suppose the corresponding theorem holds for curves of smaller genus.)

Proof. By Lemmas 7.1 and 6.3, and Proposition 3.7, we know that Theorem 1.4 holds for $(d, g)$ one of

$$
(10,9), \quad(11,10), \quad(12,12), \quad(13,13), \quad(14,14) .
$$

Similarly, by Lemmas 7.2, 6.3, and 3.5, we know that Theorem 1.6 holds for $(d, g)$ one of

$$
(16,15), \quad(17,16), \quad(18,17) .
$$

Eliminating these cases from the lists in Corollary [5.7, we obtain the given lists of pairs $(d, g)$.

Moreover - in each of the cases appearing in the statement of this corollary - results of [8] (for $r=3$ ) and [7] (for $r=4$ ) state that the Hilbert scheme of curves of degree $d$ and genus $g$ in $\mathbb{P}^{r}$ has a unique component whose points represent smooth irreducible nondegenerate curves. The condition that our curve be a BN-curve may thus be replaced with the condition that our curve be smooth irreducible nondegenerate.

\section{More Curves in a Hyperplane}

In this section, we give several more applications of the technique developed in the previous two sections. Note that from Corollary [7.3, it suffices to show the existence of curves satisfying the desired conclusions which are limits of immersions of smooth curves; it not necessary to check that these curves are BN-curves.

Lemma 8.1. Suppose $N_{f}(-2)$ satisfies interpolation, where $f: C \rightarrow \mathbb{P}^{3}$ is a general BN-curve of degree $d$ and genus $g$ to $\mathbb{P}^{3}$. Then the same is true for some smooth curve of degree and genus: 
1. $(d+3, g+3)$ (provided $d \geq 3)$;

2. $(d+4, g+2)$ (provided $d \geq 3)$;

3. $(d+4, g+6)$ (provided $d \geq 5)$.

Proof. We apply Lemma 6.3 for $f_{2}$ a curve of degree up to 4 (and note that $N_{f_{2}}(-2)$ satisfies interpolation by Proposition [3.7), namely:

1. $\left(d_{2}, g_{2}\right)=(3,1)$ and $n=3$;

2. $\left(d_{2}, g_{2}\right)=(4,0)$ and $n=3$;

3. $\left(d_{2}, g_{2}\right)=(4,2)$ and $n=5$.

Finally, we note that $C \cup_{\Gamma} D \rightarrow \mathbb{P}^{r}$ as above is a limit of immersions of smooth curves by Lemma 6.1.

Corollary 8.2. Suppose that Theorem 1.4 holds for $(d, g)=(5,1)$. Then Theorem 1.4 holds for $(d, g)$ one of:

$$
(7,2), \quad(6,3), \quad(9,6), \quad(9,7) .
$$

Proof. For $(d, g)=(7,2)$, we apply Lemma 8.1, part 2 (taking as our inductive hypothesis the truth of Theorem 1.4 for $\left.\left(d^{\prime}, g^{\prime}\right)=(3,0)\right)$.

Similarly, for $(d, g)=(6,3)$ and $(d, g)=(9,6)$, we apply Lemma 8.1, part 1 (taking as our inductive hypothesis the truth of Theorem 1.4 for $\left(d^{\prime}, g^{\prime}\right)=(3,0)$, and the just-established $\left(d^{\prime}, g^{\prime}\right)=(6,3)$, respectively).

Finally, for $(d, g)=(9,7)$, we apply Lemma 8.1, part 3 (taking as our inductive hypothesis the yet-to-be-established truth of Theorem 1.4 for $\left.\left(d^{\prime}, g^{\prime}\right)=(5,1)\right)$.

Lemma 8.3. Suppose that Theorem 1.5 holds for $(d, g)=(7,5)$. Then Theorem 1.5 holds for $(d, g)=(8,6)$.

Proof. We simply apply Lemma 2.6 with $f: C \cup_{\Gamma} D \rightarrow \mathbb{P}^{3}$ such that $\left.f\right|_{C}$ is a general BN-curve of degree 7 and genus 5 , and $\left.f\right|_{D}$ is a line, with $C$ joined to $D$ at a set $\Gamma$ of two points.

Lemma 8.4. Suppose $N_{f}(-1)$ satisfies interpolation, where $f$ is a general BN-curve of degree $d$ and genus $g$ in $\mathbb{P}^{4}$. Then the same is true for some smooth curve of degree $d+6$ and genus $g+6$, provided $d \geq 4$.

Proof. We apply Lemmas 6.3 and 6.1 for $f_{2}$ a curve of degree 6 and genus 3 to $\mathbb{P}^{3}$, with $n=4$. Note that $N_{f_{2}}(-1)$ satisfies interpolation by Propositions 3.2 and 3.3 .

Lemma 8.5. Theorem 1.6 holds for $(d, g)$ one of:

$$
(10,6), \quad(11,7), \quad(12,9) .
$$

Proof. We simply apply Lemma 8.4 (taking as our inductive hypothesis the truth of Theorem 1.6 for $\left.\left(d^{\prime}, g^{\prime}\right)=(d-6, g-6)\right)$. 
To prove the main theorems (excluding the "conversely..." part), it thus remains to produce five smooth curves:

1. For Theorem 1.4, it suffices to find smooth curves, satisfying the conclusions, of degrees and genera $(5,1),(7,4)$, and $(8,5)$.

2. For Theorem 1.5, it suffices to find a smooth curve, satisfying the conclusions, of degree 7 and genus 5 .

3. For Theorem 1.6, it suffices to find a smooth curve, satisfying the conclusions, of degree 9 and genus 5 .

\section{Curves in Del Pezzo Surfaces}

In this section, we analyze the normal bundles of certain curves by specializing to immersions $f: C \hookrightarrow \mathbb{P}^{r}$ of smooth curves whose images are contained in Del Pezzo surfaces $S \subset \mathbb{P}^{r}$ (where the Del Pezzo surface is embedded by its complete anticanonical series). Since $f$ will be an immersion, we shall identify $C=f(C)$ with its image, in which case the normal bundle $N_{f}$ becomes the normal bundle $N_{C}$ of the image. Our basic method in this section will be to use the normal bundle exact sequence associated to $C \subset S \subset \mathbb{P}^{r}$ :

$$
\left.0 \rightarrow N_{C / S} \rightarrow N_{C} \rightarrow N_{S}\right|_{C} \rightarrow 0 .
$$

Since $S$ is a Del Pezzo surface, we have by adjunction an isomorphism

$$
N_{C / S} \simeq K_{C} \otimes K_{S}^{\vee} \simeq K_{C}(1) .
$$

Definition 9.1. Let $S \subset \mathbb{P}^{r}$ be a Del Pezzo surface, $k$ be an integer with $H^{1}\left(N_{S}(-k)\right)=0$, and $\theta \in \operatorname{Pic} S$ be any divisor class.

Let $F$ be a general hypersurface of degree $k$. We consider the moduli space $\mathcal{M}$ of pairs $\left(S^{\prime}, \theta^{\prime}\right)$, with $S^{\prime}$ a Del Pezzo surface containing $S \cap F$, and $\theta^{\prime} \in \operatorname{Pic} S^{\prime}$. Define $V_{\theta, k} \subseteq \operatorname{Pic}(S \cap F)$ to be the subvariety obtained by restricting $\theta^{\prime}$ to $S \cap F \subseteq S^{\prime}$, as $\left(S^{\prime}, \theta\right)$ varies over the component of $\mathcal{M}$ containing $(S, \theta)$.

Note that there is a unique such component, since $\mathcal{M}$ is smooth at $[(S, \theta)]$ thanks to our assumption that $H^{1}\left(N_{S}(-k)\right)=0$.

Our essential tool is given by the following lemma, which uses the above normal bundle sequence together with the varieties $V_{\theta, k}$ to analyze $N_{C}$.

Lemma 9.2. Let $C \subset S \subset \mathbb{P}^{r}$ be a general curve (of any fixed class) in a general Del Pezzo surface $S \subset \mathbb{P}^{r}$, and $k$ be a natural number with $H^{1}\left(N_{S}(-k)\right)=0$. Suppose that (for $F$ a general hypersurface of degree $k$ ):

$$
\operatorname{dim} V_{[C], k}=\operatorname{dim} H^{0}\left(\mathcal{O}_{C}(k-1)\right) \quad \text { and } \quad H^{1}\left(\left.N_{S}\right|_{C}(-k)\right)=0,
$$

and that the natural map

$$
H^{0}\left(N_{S}(-k)\right) \rightarrow H^{0}\left(\left.N_{S}\right|_{C}(-k)\right)
$$

is an isomorphism. Then,

$$
H^{1}\left(N_{C}(-k)\right)=0
$$


Proof. Twisting our earlier normal bundle exact sequence (3), and using the isomorphism (4), we obtain the exact sequence:

$$
\left.0 \rightarrow K_{C}(1-k) \rightarrow N_{C}(-k) \rightarrow N_{S}\right|_{C}(-k) \rightarrow 0
$$

This gives rise to a long exact sequence in cohomology:

$$
\cdots \rightarrow H^{0}\left(N_{C}(-k)\right) \rightarrow H^{0}\left(\left.N_{S}\right|_{C}(-k)\right) \rightarrow H^{1}\left(K_{C}(1-k)\right) \rightarrow H^{1}\left(N_{C}(-k)\right) \rightarrow H^{1}\left(\left.N_{S}\right|_{C}(-k)\right) \rightarrow \cdots
$$

Since $H^{1}\left(\left.N_{S}\right|_{C}(-k)\right)=0$ by assumption, it suffices to show that the image of the natural map $H^{0}\left(N_{C}(-k)\right) \rightarrow H^{0}\left(\left.N_{S}\right|_{C}(-k)\right)$ has codimension

$$
\operatorname{dim} H^{1}\left(K_{C}(1-k)\right)=\operatorname{dim} H^{0}\left(\mathcal{O}_{C}(k-1)\right)=\operatorname{dim} V_{[C], k} .
$$

Because the natural map $H^{0}\left(N_{S}(-k)\right) \rightarrow H^{0}\left(\left.N_{S}\right|_{C}(-k)\right)$ is an isomorphism, we may interpret sections of $\left.N_{S}\right|_{C}(-k)$ as first-order deformations of the Del Pezzo surface $S$ fixing $S \cap F$. So it remains to show that the space of such deformations coming from a deformation of $C$ fixing $C \cap F$ has codimension $\operatorname{dim} V_{[C], k}$.

The key point here is that deforming $C$ on $S$ does not change its class $[C] \in \operatorname{Pic}(S)$, and every deformation of $S$ comes naturally with a deformation of the element $[C] \in \operatorname{Pic}(S)$. It thus suffices to prove that the space of first-order deformations of $S$ which leave invariant the restriction $\left.[C]\right|_{S \cap F} \in \operatorname{Pic}(S \cap F)$ has codimension $\operatorname{dim} V_{[C], k}$.

But since the map $\mathcal{M} \rightarrow V_{[C], k}$ is smooth at $(S,[C])$, the vertical tangent space has codimension in the full tangent space equal to the dimension of the image.

In applying Lemma 9.2, we will first consider the case where $S \subset \mathbb{P}^{3}$ is a general cubic surface, which is isomorphic to the blowup $\mathrm{Bl}_{\Gamma} \mathbb{P}^{2}$ of $\mathbb{P}^{2}$ along a set

$$
\Gamma=\left\{p_{1}, \ldots, p_{6}\right\} \subset \mathbb{P}^{2}
$$

of six general points. Recall that this a Del Pezzo surface, which is to say that the embedding $\mathrm{Bl}_{\Gamma} \mathbb{P}^{2} \simeq S \hookrightarrow \mathbb{P}^{3}$ as a cubic surface is via the complete linear system for the inverse of the canonical bundle:

$$
-K_{\mathrm{Bl}_{\Gamma} \mathbb{P}^{2}}=3 L-E_{1}-\cdots-E_{6},
$$

where $L$ is the class of a line in $\mathbb{P}^{2}$ and $E_{i}$ is the exceptional divisor in the blowup over $p_{i}$. Note that by construction,

$$
N_{S} \simeq \mathcal{O}_{S}(3)
$$

In particular, $H^{1}\left(N_{S}(-1)\right)=H^{1}\left(N_{S}(-2)\right)=0$ by Kodaira vanishing.

Lemma 9.3. Let $C \subset \mathrm{Bl}_{\Gamma} \mathbb{P}^{2} \simeq S \subset \mathbb{P}^{3}$ be a general curve of class either:

1. $5 L-2 E_{1}-2 E_{2}-E_{3}-E_{4}-E_{5}-E_{6}$;

2. $5 L-2 E_{1}-E_{2}-E_{3}-E_{4}-E_{5}-E_{6}$;

3. $6 L-E_{1}-E_{2}-2 E_{3}-2 E_{4}-2 E_{5}-2 E_{6}$;

4. $6 L-E_{1}-2 E_{2}-2 E_{3}-2 E_{4}-2 E_{5}-2 E_{6}$; 
Then $C$ is smooth and irreducible. In the first two cases, $H^{1}\left(\mathcal{O}_{C}(1)\right)=0$.

Proof. We first show the above linear series are basepoint-free. To do this, we write each as a sum of terms which are evidently basepoint-free:

$$
\begin{aligned}
5 L-2 E_{1}-2 E_{2}-E_{3}-E_{4}-E_{5}-E_{6}= & \left(3 L-E_{1}-E_{2}-E_{3}-E_{4}-E_{5}-E_{6}\right) \\
& +\left(L-E_{1}\right)+\left(L-E_{2}\right) \\
5 L-2 E_{1}-E_{2}-E_{3}-E_{4}-E_{5}-E_{6}= & \left(3 L-E_{1}-E_{2}-E_{3}-E_{4}-E_{5}-E_{6}\right)+\left(L-E_{1}\right) \\
6 L-E_{1}-E_{2}-2 E_{3}-2 E_{4}-2 E_{5}-2 E_{6}= & \left(3 L-E_{1}-E_{2}-E_{3}-E_{4}-E_{5}-E_{6}\right) \\
& +L+\left(2 L-E_{3}-E_{4}-E_{5}-E_{6}\right) \\
6 L-E_{1}-2 E_{2}-2 E_{3}-2 E_{4}-2 E_{5}-2 E_{6}= & \left(3 L-E_{1}-E_{2}-E_{3}-E_{4}-E_{5}-E_{6}\right) \\
& +\left(L-E_{2}\right)+\left(2 L-E_{3}-E_{4}-E_{5}-E_{6}\right) . \\
&
\end{aligned}
$$

Since all our linear series are basepoint-free, the Bertini theorem implies that $C$ is smooth. Moreover, by basepoint-freeness, we know that $C$ does not contain any of our exceptional divisors. We conclude that $C$ is a the proper transform in the blowup of a curve $C_{0} \subset \mathbb{P}^{2}$. This curve satisfies:

- In case 1, $C_{0}$ has exactly two nodes, at $p_{1}$ and $p_{2}$, and is otherwise smooth. In particular, $C_{0}$ (and thus $C$ ) must be irreducible, since otherwise (by Bézout's theorem) it would have at least 4 nodes (where the components meet).

- In case 2, $C_{0}$ has exactly one node, at $p_{1}$, and is otherwise smooth. As above, $C_{0}$ (and thus $C$ ) must be irreducible.

- In case 3, $C_{0}$ has exactly four nodes, at $\left\{p_{3}, p_{4}, p_{5}, p_{6}\right\}$, and is otherwise smooth. As above, $C_{0}$ (and thus $C$ ) must be irreducible.

- In case 4, $C_{0}$ has exactly 5 nodes, at $\left\{p_{2}, p_{3}, p_{4}, p_{5}, p_{6}\right\}$, and is otherwise smooth. As above, $C_{0}$ must either be irreducible, or the union of a line and a quintic. (Otherwise, it would have at least 8 nodes.) But in the second case, all 5 nodes must be collinear, contradicting our assumption that $\left\{p_{2}, p_{3}, p_{4}, p_{5}, p_{6}\right\}$ are general. Consequently, $C_{0}$ (and thus $C$ ) must be irreducible.

We now turn to showing $H^{1}\left(\mathcal{O}_{C}(1)\right)=0$ in the first two cases. In the first case, we note that $\Gamma$ contains $4=\operatorname{genus}(C)$ general points $\left\{p_{3}, p_{4}, p_{5}, p_{6}\right\}$ on $C$; consequently, $E_{3}+E_{4}+E_{5}+E_{6}$ - and therefore $\mathcal{O}_{C}(1)=\left(3 L-E_{1}-E_{2}\right)-\left(E_{3}+E_{4}+E_{5}+E_{6}\right)$ - is a general line bundle of degree 7 , which implies $H^{1}\left(\mathcal{O}_{C}(1)\right)=0$. Similarly, in the second case, we note that $\Gamma$ contains $5=\operatorname{genus}(C)$ general points $\left\{p_{2}, p_{3}, p_{4}, p_{5}, p_{6}\right\}$ on $C$. As in the first case, this implies $H^{1}\left(\mathcal{O}_{C}(1)\right)=0$, as desired.

Lemma 9.4. Let $C \subset \mathbb{P}^{3}$ be a general $B N$-curve of degree and genus $(7,4)$ or $(8,5)$. Then we have $H^{1}\left(N_{C}(-2)\right)=0$.

Proof. We take $C \subset S$, as constructed in Lemma 9.3 , parts 1 and 2 respectively. These curves have degrees and genera $(7,4)$ and $(8,5)$ respectively, which can be seen by calculating the 
intersection product with the hyperplane class and using adjunction. For example, for the curve in part 1 of class $5 L-2 E_{1}-2 E_{2}-E_{3}-E_{4}-E_{5}-E_{6}$, we calculate

$$
\operatorname{deg} C=\left(5 L-2 E_{1}-2 E_{2}-E_{3}-E_{4}-E_{5}-E_{6}\right) \cdot\left(3 L-E_{1}-E_{2}-E_{3}-E_{4}-E_{5}-E_{6}\right)=7,
$$

and

$$
\text { genus } C=1+\frac{K_{S} \cdot C+C^{2}}{2}=1+\frac{-\operatorname{deg} C+C^{2}}{2}=1+\frac{-7+13}{2}=4 .
$$

Because $N_{S} \simeq \mathcal{O}_{S}(3)$, we have

$$
H^{1}\left(\left.N_{S}\right|_{C}(-2)\right)=H^{1}\left(\mathcal{O}_{C}(1)\right)=0 .
$$

Moreover, $\mathcal{O}_{S}(1)(-C)$ is either $-2 L+E_{1}+E_{2}$ or $-2 L+E_{1}$ respectively; in either case we have $H^{0}\left(\mathcal{O}_{S}(1)(-C)\right)=0$. Consequently, the restriction map

$$
H^{0}\left(\mathcal{O}_{S}(1)\right) \rightarrow H^{0}\left(\mathcal{O}_{C}(1)\right)
$$

is injective. Since

$$
\operatorname{dim} H^{0}\left(\mathcal{O}_{S}(1)\right)=4=\operatorname{dim} H^{0}\left(\mathcal{O}_{C}(1)\right)
$$

the above restriction map is therefore an isomorphism. Applying Lemma 9.2, it thus suffices to show that

$$
\operatorname{dim} V_{[C], 2}=\operatorname{dim} H^{0}\left(\mathcal{O}_{C}(1)\right)=4
$$

To do this, we first observe that $[C]$ is always a linear combination $a H+b L_{1}+c L_{2}$ of the hyperplane class $H$, and two nonintersecting lines $L_{1}$ and $L_{2}$, such that both $b$ and $c$ are nonvanishing. Indeed:

$$
\begin{aligned}
5 L-2 E_{1}-2 E_{2}-E_{3}-E_{4}-E_{5}-E_{6}= & 3\left(3 L-E_{1}-E_{2}-E_{3}-E_{4}-E_{5}-E_{6}\right) \\
& -\left(2 L-E_{1}-E_{3}-E_{4}-E_{5}-E_{6}\right) \\
& -\left(2 L-E_{2}-E_{3}-E_{4}-E_{5}-E_{6}\right) \\
5 L-2 E_{1}-E_{2}-E_{3}-E_{4}-E_{5}-E_{6}= & 3\left(3 L-E_{1}-E_{2}-E_{3}-E_{4}-E_{5}-E_{6}\right)+E_{1} \\
& -2\left(2 L-E_{2}-E_{3}-E_{4}-E_{5}-E_{6}\right) .
\end{aligned}
$$

Writing $F$ for a general quadric hypersurface, and $D=F \cap S$, we observe that $\operatorname{Pic}(D)$ is 4-dimensional. It is therefore sufficient to prove that for a general class $\theta \in \operatorname{Pic}^{6 a+2 b+2 c}(D)$, there exists a smooth cubic surface $S$ containing $D$ and a pair $\left(L_{1}, L_{2}\right)$ of disjoint lines on $S$, such that the restriction $\left.\left(a H+b L_{1}+c L_{2}\right)\right|_{D}=\theta$. Since $\left.H\right|_{D}=\mathcal{O}_{D}(1)$ is independent of $S$ and the choice of $\left(L_{1}, L_{2}\right)$, we may replace $\theta$ by $\theta(-a)$ and set $a=0$.

We thus seek to show that for $b, c \neq 0$ and $\theta \in \operatorname{Pic}^{2 b+2 c}(D)$ general, there exists a smooth cubic surface $S$ containing $D$, and a pair $\left(L_{1}, L_{2}\right)$ of disjoint lines on $S$, with $\left.\left(b L_{1}+c L_{2}\right)\right|_{D}=\theta$. Equivalently, we want to show the map

$$
\left\{\left(S, E_{1}, E_{2}\right): E_{1}, E_{2} \subset S \supset D\right\} \mapsto\left\{\left(E_{1}, E_{2}\right)\right\}
$$

from the space of smooth cubic surfaces $S$ containing $D$ with a choice of pair of disjoint lines $\left(E_{1}, E_{2}\right)$, to the space of pairs of 2 -secant lines to $D$, is dominant. For this, it suffices to check the vanishing of $H^{1}\left(N_{S}\left(-D-E_{1}-E_{2}\right)\right)$, for any smooth cubic $S$ containing $D$ and disjoint lines $\left(E_{1}, E_{2}\right)$ on $S$, in which lies the obstruction to smoothness of this map. But $N_{S}\left(-D-E_{1}-E_{2}\right)=3 L-2 E_{1}-2 E_{2}-E_{3}-E_{4}-E_{5}-E_{6}$ has no higher cohomology by Kawamata-Viehweg vanishing. 
Lemma 9.5. Let $C \subset \mathbb{P}^{3}$ be a general $B N$-curve of degree 7 and genus 5 . Then we have $H^{1}\left(N_{C}(-1)\right)=0$.

Proof. We take $C \subset S$, as constructed in Lemma 9.3, part 4. Because $N_{S} \simeq \mathcal{O}_{S}(3)$, we have

$$
H^{1}\left(\left.N_{S}\right|_{C}(-1)\right)=H^{1}\left(\mathcal{O}_{C}(2)\right)=0 .
$$

Moreover, $\mathcal{O}_{S}(2)(-C) \simeq \mathcal{O}_{S}\left(-E_{1}\right)$ has no sections. Consequently, the restriction map

$$
H^{0}\left(\mathcal{O}_{S}(2)\right) \rightarrow H^{0}\left(\mathcal{O}_{C}(2)\right)
$$

is injective. Since

$$
\operatorname{dim} H^{0}\left(\mathcal{O}_{S}(2)\right)=10=\operatorname{dim} H^{0}\left(\mathcal{O}_{C}(2)\right),
$$

the above restriction map is therefore an isomorphism. Applying Lemma 9.2, it thus suffices to show that

$$
\operatorname{dim} V_{[C], 1}=\operatorname{dim} H^{0}\left(\mathcal{O}_{C}\right)=1
$$

Writing $F$ for a general hyperplane, and $D=F \cap S$, we observe that $\operatorname{Pic}(D)$ is 1-dimensional. Since $[C]=2 H+E_{1}$, it is therefore sufficient to prove that for a general class $\theta \in \operatorname{Pic}^{7}(D)$, there exists a cubic surface $S$ containing $D$ and a line $L$ on $S$, such that the restriction $\left.(2 H+L)\right|_{D}=\theta$. Since $\left.H\right|_{D}=\mathcal{O}_{D}(1)$ is independent of $S$ and the choice of $L$, we may replace $\theta$ by $\theta(-1)$ and look instead for $\left.L\right|_{D}=\theta \in \operatorname{Pic}^{1}(D)$. Equivalently, we want to show the map

$$
\left\{\left(S, E_{1}\right): E_{1} \subset S \supset D\right\} \mapsto\left\{\left(E_{1}, E_{2}\right)\right\}
$$

from the space of smooth cubic surfaces $S$ containing $D$ with a choice of line $E_{1}$, to the space of 1-secant lines to $D$, is dominant; it suffices to check the vanishing of $H^{1}\left(N_{S}\left(-D-E_{1}\right)\right)$, for any smooth cubic $S$ containing $D$ and line $E_{1}$ on $S$, in which lies the obstruction to smoothness of this map. But $N_{S}\left(-D-E_{1}\right)=6 L-3 E_{1}-2 E_{2}-2 E_{3}-2 E_{4}-2 E_{5}-2 E_{6}$ has no higher cohomology by Kodaira vanishing.

Next, we consider the case where $S \subset \mathbb{P}^{4}$ is the intersection of two quadrics, which is isomorphic to the blowup $\mathrm{Bl}_{\Gamma} \mathbb{P}^{2}$ of $\mathbb{P}^{2}$ along a set

$$
\Gamma=\left\{p_{1}, \ldots, p_{5}\right\}
$$

of five general points. Recall that this is a Del Pezzo surface, which is to say that the embedding $\mathrm{Bl}_{\Gamma} \mathbb{P}^{2} \simeq S \hookrightarrow \mathbb{P}^{4}$ as the intersection of two quadrics is via the complete linear system for the inverse of the canonical bundle:

$$
-K_{\mathrm{Bl}_{\Gamma} \mathbb{P}^{2}}=3 L-E_{1}-\cdots-E_{5},
$$

where $L$ is the class of a line in $\mathbb{P}^{2}$ and $E_{i}$ is the exceptional divisor in the blowup over $p_{i}$. Note that by construction,

$$
N_{S} \simeq \mathcal{O}_{S}(2) \oplus \mathcal{O}_{S}(2)
$$

In particular, $H^{1}\left(N_{S}(-1)\right)=0$ by Kodaira vanishing.

Lemma 9.6. Let $C \subset \mathrm{Bl}_{\Gamma} \mathbb{P}^{2} \simeq S \subset \mathbb{P}^{4}$ be a general curve of class either: 
1. $5 L-2 E_{1}-E_{2}-E_{3}-E_{4}-E_{5}$;

2. $6 L-E_{1}-2 E_{2}-2 E_{3}-2 E_{4}-2 E_{5}$.

Then $C$ is smooth and irreducible. In the first case, $H^{1}\left(\mathcal{O}_{C}(1)\right)=0$.

Proof. We first show the above linear series are basepoint-free. To do this, we write them as a sum of terms which are evidently basepoint-free:

$$
\begin{aligned}
5 L-2 E_{1}-E_{2}-E_{3}-E_{4}-E_{5}= & \left(3 L-E_{1}-E_{2}-E_{3}-E_{4}-E_{5}\right)+\left(L-E_{1}\right)+L \\
6 L-E_{1}-2 E_{2}-2 E_{3}-2 E_{4}-2 E_{5}=\left(3 L-E_{1}-E_{2}-E_{3}-E_{4}-E_{5}\right) & \\
& +\left(2 L-E_{2}-E_{3}-E_{4}-E_{5}\right)+L
\end{aligned}
$$

As in Lemma 9.3, we conclude that $C$ is smooth and irreducible. In the first case, we have $\operatorname{deg} \mathcal{O}_{C}(1)=9>8=2 g-2$, which implies $H^{1}\left(\mathcal{O}_{C}(1)\right)=0$ as desired.

Lemma 9.7. Let $C \subset \mathbb{P}^{4}$ be a general $B N$-curve of degree 9 and genus 5 . Then we have $H^{1}\left(N_{C}(-1)\right)=0$.

Proof. We take $C \subset S$, as constructed in Lemma 9.6. Because $N_{S} \simeq \mathcal{O}_{S}(2) \oplus \mathcal{O}_{S}(2)$, we have

$$
H^{1}\left(\left.N_{S}\right|_{C}(-1)\right)=H^{1}\left(\mathcal{O}_{C}(1) \oplus \mathcal{O}_{C}(1)\right)=0 .
$$

Moreover, $\mathcal{O}_{S}(1)(-C) \simeq \mathcal{O}_{S}\left(-2 L+E_{1}\right)$ has no sections. Consequently, the restriction map

$$
H^{0}\left(\mathcal{O}_{S}(1) \oplus \mathcal{O}_{S}(1)\right) \rightarrow H^{0}\left(\mathcal{O}_{C}(1) \oplus \mathcal{O}_{C}(1)\right)
$$

is injective. Since

$$
\operatorname{dim} H^{0}\left(\mathcal{O}_{S}(1) \oplus \mathcal{O}_{S}(1)\right)=10=\operatorname{dim} H^{0}\left(\mathcal{O}_{C}(1) \oplus \mathcal{O}_{C}(1)\right),
$$

the above restriction map is therefore an isomorphism. Applying Lemma 9.2, it thus suffices to show that

$$
\operatorname{dim} V_{[C], 1}=\operatorname{dim} H^{0}\left(\mathcal{O}_{C}\right)=1 .
$$

Writing $F$ for a general hyperplane, and $D=F \cap S$, we observe that $\operatorname{Pic}(D)$ is 1-dimensional. Since $[C]=3\left(3 L-E_{1}-E_{2}-E_{3}-E_{4}-E_{5}\right)-2\left(2 L-E_{1}-E_{2}-E_{3}-E_{4}-E_{5}\right)-E_{1}$, it is therefore sufficient to prove that for a general class $\theta \in \operatorname{Pic}^{9}(D)$, there exists a quartic Del Pezzo surface $S$ containing $D$, and a pair $\left\{L_{1}, L_{2}\right\}$ of intersecting lines on $S$, such that the restriction $\left.\left(3 H-2 L_{1}-L_{2}\right)\right|_{D}=\theta$. Since $\left.H\right|_{D}=\mathcal{O}_{D}(1)$ is independent of $S$ and the choice of $L$, we may replace $\theta$ by $\theta^{-1}(3)$ and look instead for $\left.\left(2 L_{1}+L_{2}\right)\right|_{D}=\theta \in \operatorname{Pic}^{3}(D)$. For this, it suffices to show the map

$$
\left\{\left(S, L_{1}, L_{2}\right): L_{1}, L_{2} \subset S \supset D\right\} \mapsto\left\{\left(L_{1}, L_{2}\right)\right\},
$$

from the space of smooth quartic Del Pezzo surfaces $S$ containing $D$ with a choice of pair of intersecting lines $\left(L_{1}, L_{2}\right)$, to the space of pairs of intersecting 1-secant lines to $D$, is dominant. Taking $\left[L_{1}\right]=E_{1}$ and $\left[L_{2}\right]=L-E_{1}-E_{2}$, it suffices to check the vanishing of the first cohomology of the vector bundle $N_{S}\left(-D-E_{1}-\left(L-E_{1}-E_{2}\right)\right)$ - which is isomorphic to a direct sum of two copies of the line bundle $2 L-E_{1}-E_{3}-E_{4}-E_{5}$ - for any smooth quartic Del Pezzo surface $S$ containing $D$, in which lies the obstruction to smoothness of this map. But $2 L-E_{1}-E_{3}-E_{4}-E_{5}$ has no higher cohomology by Kodaira vanishing.

To prove the main theorems (excluding the "conversely..." part), it thus remains to produce a smooth curve $C \subset \mathbb{P}^{3}$ of degree 5 and genus 1 , with $H^{1}\left(N_{C}(-2)\right)=0$. 


\section{Elliptic Curves of Degree 5 in $\mathbb{P}^{3}$}

In this section, we construct an immersion $f: C \hookrightarrow \mathbb{P}^{3}$ of degree 5 from a smooth elliptic curve, with $H^{1}\left(N_{f}(-2)\right)=0$. As in the previous section, we shall identify $C=f(C)$ with its image, in which case the normal bundle $N_{f}$ becomes the normal bundle $N_{C}$ of the image.

Our basic method in this section will be to use the geometry of the cubic scroll $S \subset \mathbb{P}^{4}$. Recall that the cubic scroll can be constructed in two different ways:

1. Let $Q \subset \mathbb{P}^{4}$ and $M \subset \mathbb{P}^{4}$ be a plane conic, and a line disjoint from the span of $Q$, respectively. As abstract varieties, $Q \simeq \mathbb{P}^{1} \simeq M$. Then $S$ is the ruled surface swept out by lines joining pairs of points identified under some choice of above isomorphism.

2. Let $x \in \mathbb{P}^{2}$ be a point, and consider the blowup $\mathrm{Bl}_{x} \mathbb{P}^{2}$ of $\mathbb{P}^{2}$ at the point $\{x\}$. Then, $S$ is the image of $f: \mathrm{Bl}_{x} \mathbb{P}^{2} \hookrightarrow \mathbb{P}^{4}$ under the complete linear series attached to the line bundle

$$
2 L-E
$$

where $L$ is the class of a line in $\mathbb{P}^{2}$, and $E$ is the exceptional divisor in the blowup.

To relate these two constructions, we fix a line $L \subset \mathbb{P}^{2}$ not meeting $x$ in the second construction, and consider the isomorphism $L \simeq \mathbb{P}^{1} \simeq E$ defined by sending $p \in L$ to the intersection with $E$ of the proper transform of the line joining $p$ and $x$. Then the $f(L)$ and $f(E)$ are $Q$ and $M$ respectively in the second construction; the proper transforms of lines through $x$ are the lines of the ruling.

Now take two points $p, q \in L$. Since $f(L)$ is a plane conic, the tangent lines to $f(L)$ at $p$ and $q$ intersect; we let $y$ be their point of intersection.

From the first description of $S$, it is clear that any line through $y$ intersects $S$ quasitransversely - except for the lines joining $y$ to $p$ and $q$, each of which meets $S$ in a degree 2 subscheme of $f(L)$. Write $\bar{S}$ for the image of $S$ under projection from $y$; by construction, the projection $\pi: S \rightarrow \bar{S} \subseteq \mathbb{P}^{3}$ is unramified away from $\{p, q\}$, an immersion away from $f(L)$, and when restricted to $f(L)$ is a double cover of its image with ramification exactly at $\{p, q\}$. At $\{p, q\}$, the differential drops rank transversely, with kernel the tangent space to $f(L)$. (By "drops rank transversely", we mean that the section $d \pi$ of $\operatorname{Hom}\left(T_{S}, \pi^{*} T_{\mathbb{P}^{3}}\right)$ is transverse to the subvariety of $\operatorname{Hom}\left(T_{S}, \pi^{*} T_{\mathbb{P}^{3}}\right)$ of maps with less-than-maximal rank.)

If $C \subset \mathrm{Bl}_{\{p\}} \mathbb{P}^{2} \simeq S$ is a curve passing through $p$ and $q$, but transverse to $L$ at each of these points, then any line through $y$ intersects $C$ quasi-transversely. In particular, if $C$ meets $L$ in at most one point outside of $\{p, q\}$, the image $\bar{C}$ of $C$ under projection from $y$ is smooth. Moreover, the above analysis of $d \pi$ on $S$ implies that the natural map

$$
N_{C / S} \rightarrow N_{\bar{C} / \mathbb{P}^{3}}
$$

induced by $\pi$ is fiberwise injective away from $\{p, q\}$, and has a simple zero at both $p$ and $q$. That is, we have an exact sequence

$$
0 \rightarrow N_{C / S}(p+q) \rightarrow N_{\bar{C} / \mathbb{P}^{3}} \rightarrow \mathcal{Q} \rightarrow 0
$$

with $\mathcal{Q}$ a vector bundle. 
We now specialize to the case where $C$ is the proper transform of a plane cubic, passing through $\{x, p, q\}$, and transverse to $L$ at $\{p, q\}$. By inspection, $\bar{C}$ is an elliptic curve of degree 5 in $\mathbb{P}^{3}$; it thus suffices to show $H^{1}\left(N_{\bar{C} / \mathbb{P}^{3}}(-2)\right)=0$.

Lemma 10.1. In this case,

$$
\begin{aligned}
N_{C / S}(p+q) & \simeq \mathcal{O}_{C}(3 L-E+p+q) \\
\mathcal{Q} & \simeq \mathcal{O}_{C}(5 L-3 E-p-q) .
\end{aligned}
$$

Proof. We first note that

$$
N_{C / S} \simeq N_{C / \mathbb{P}^{2}}(-E) \simeq \mathcal{O}_{C}(3 L)(-E) \quad \Rightarrow \quad N_{C / S}(p+q) \simeq \mathcal{O}_{C}(3 L-E+p+q) .
$$

Next, the Euler exact sequence

$$
\left.0 \rightarrow \mathcal{O}_{\bar{C}} \rightarrow \mathcal{O}_{\bar{C}}(1)^{4} \rightarrow T_{\mathbb{P}^{3}}\right|_{\bar{C}} \rightarrow 0
$$

implies

$$
\wedge^{3}\left(\left.T_{\mathbb{P}^{3}}\right|_{\bar{C}}\right) \simeq \mathcal{O}_{C}(4)
$$

Combined with the normal bundle exact sequence

$$
\left.0 \rightarrow T_{C} \rightarrow T_{\mathbb{P}^{3}}\right|_{\bar{C}} \rightarrow N_{\bar{C} / \mathbb{P}^{3}} \rightarrow 0,
$$

and the fact that $C$ is of genus 1 , so $T_{C} \simeq \mathcal{O}_{C}$, we conclude that

$$
\wedge^{2}\left(N_{\bar{C} / \mathbb{P}^{3}}\right) \simeq \mathcal{O}_{C}(4) \otimes T_{C}^{\vee} \simeq \mathcal{O}_{C}(4)=\mathcal{O}_{C}(4(2 L-E))=\mathcal{O}_{C}(8 L-4 E)
$$

The exact sequence (5) then implies

$$
\mathcal{Q} \simeq \wedge^{2}\left(N_{\bar{C} / \mathbb{P}^{3}}\right) \otimes\left(N_{C / S}(p+q)\right)^{\vee} \simeq \mathcal{O}_{C}(8 L-4 E)(-3 L+E-p-q)=\mathcal{O}_{C}(5 L-3 E-p-q),
$$

as desired.

Twisting by $\mathcal{O}_{C}(-2) \simeq \mathcal{O}_{C}(-4 L+2 E)$, we obtain isomorphisms:

$$
\begin{aligned}
N_{C / S}(p+q) & \simeq \mathcal{O}_{C}(-L+E+p+q) \\
\mathcal{Q} & \simeq \mathcal{O}_{C}(L-E-p-q) .
\end{aligned}
$$

We thus have an exact sequence

$$
0 \rightarrow \mathcal{O}_{C}(-L+E+p+q) \rightarrow N_{\bar{C} / \mathbb{P}^{3}}(-2) \rightarrow \mathcal{O}_{C}(L-E-p-q) \rightarrow 0 .
$$

Since $\mathcal{O}_{C}(-L+E+p+q)$ and $\mathcal{O}_{C}(L-E-p-q)$ are both general line bundles of degree zero on a curve of genus 1 , we have

$$
H^{1}\left(\mathcal{O}_{C}(-L+E+p+q)\right)=H^{1}\left(\mathcal{O}_{C}(L-E-p-q)\right)=0
$$

which implies

$$
H^{1}\left(N_{\bar{C} / \mathbb{P}^{3}}(-2)\right)=0 .
$$

This completes the proof the main theorems, except for the "conversely..." parts. 


\section{The Converses}

In this section, we show that the intersections appearing in our main theorems fail to be general in all listed exceptional cases. We actually go further, describing precisely the intersection of a general BN-curve $f: C \rightarrow \mathbb{P}^{r}$ in terms of the intrinsic geometry of $Q \simeq \mathbb{P}^{1} \times \mathbb{P}^{1}, H \simeq \mathbb{P}^{2}$, and $H \simeq \mathbb{P}^{3}$ respectively.

Since the general BN-curve $f: C \rightarrow \mathbb{P}^{r}$ is an immersion, we can identify $C=f(C)$ with its image as in the previous two sections, in which case the normal bundle $N_{f}$ becomes the normal bundle $N_{C}$ of its image.

There are two basic phenomenon which occur explain the majority of our exceptional cases: cases where $C$ is a complete intersection, and cases where $C$ lies on a surface of low degree. The first two subsections will be devoted to the exceptional cases that arise for these two reasons respectively. In the final subsection, we will consider the two remaining exceptional cases.

\subsection{Complete Intersections}

We begin by dealing with those exceptional cases which are complete intersections.

Proposition 11.1. Let $C \subset \mathbb{P}^{3}$ be a general $B N$-curve of degree 4 and genus 1 . Then the intersection $C \cap Q$ is the intersection of two general curves of bidegree $(2,2)$ on $Q \simeq \mathbb{P}^{1} \times \mathbb{P}^{1}$. In particular, it is not a collection of 8 general points.

Proof. It is easy to see that $C$ is the complete intersection of two general quadrics. Restricting these quadrics to $Q \simeq \mathbb{P}^{1} \times \mathbb{P}^{1}$, we see that $C \cap Q$ is the intersection of two general curves of bidegree $(2,2)$.

Since general points impose independent conditions on the 9-dimensional space of curves of bidegree $(2,2)$, a general collection of 8 points will lie only on one curve of bidegree $(2,2)$. The intersections of two general curves of bidegree $(2,2)$ is therefore not a collection of 8 general points.

Proposition 11.2. Let $C \subset \mathbb{P}^{3}$ be a general BN-curve of degree 6 and genus 4 . Then the intersection $C \cap Q$ is the intersection of two general curves of bidegrees $(2,2)$ and $(3,3)$ respectively on $Q \simeq \mathbb{P}^{1} \times \mathbb{P}^{1}$. In particular, it is not a collection of 12 general points.

Proof. It is easy to see that $C$ is the complete intersection of a general quadric and cubic. Restricting these to $Q \simeq \mathbb{P}^{1} \times \mathbb{P}^{1}$, we see that $C \cap Q$ is the intersection of two general curves of bidegrees $(2,2)$ and $(3,3)$ respectively.

Since general points impose independent conditions on the 9-dimensional space of curves of bidegree $(2,2)$, a general collection of 12 points will not lie any curve of bidegree $(2,2)$, and in particular will not be such an intersection.

Proposition 11.3. Let $C \subset \mathbb{P}^{3}$ be a general $B N$-curve of degree 6 and genus 4 . Then the intersection $C \cap H$ is a general collection of 6 points lying on a conic. In particular, it is not a collection of 6 general points.

Proof. As in Proposition 11.2, we see that $C \cap H$ is the intersection of general conic and cubic curves. 
In particular, $C \cap H$ lies on a conic. Conversely, any 6 points lying on a conic are the complete intersection of a conic and a cubic by Theorem $1.2($ with $(d, g)=(3,1)$ ).

Since general points impose independent conditions on the 6-dimensional space of plane conics, a general collection of 6 points will not lie on a conic. We thus see our intersection is not a collection of 6 general points.

Proposition 11.4. Let $C \subset \mathbb{P}^{4}$ be a general $B N$-curve of degree 8 and genus 5 . Then the intersection $C \cap H$ is the intersection of three general quadrics in $H \simeq \mathbb{P}^{3}$. In particular, it is not a collection of 8 general points.

Proof. It is easy to see that $C$ is the complete intersection of three general quadrics. Restricting these quadrics to $H \simeq \mathbb{P}^{3}$, we see that $C \cap H$ is the intersection of three general quadrics.

Since general points impose independent conditions on the 10-dimensional space of quadrics, a general collection of 8 points will lie only on only two quadrics. The intersection of three general quadrics is therefore not a collection of 8 general points.

\subsection{Curves on Surfaces}

Next, we analyze those cases which are exceptional because $C$ lies on a surface $S$ of small degree. To show the intersection is general subject to the constraint imposed by $C \subset S$, it will be useful to have the following lemma:

Lemma 11.5. Let $D$ be an irreducible curve of genus $g$ on a surface $S$, and $p_{1}, p_{2}, \ldots, p_{n}$ be a collection of $n$ distinct points on $D$. Suppose that $n \geq g$, and that $p_{1}, p_{2}, \ldots, p_{g}$ are general. Let $\theta \in \operatorname{Pic}(S)$, with $\left.\theta\right|_{D} \sim p_{1}+p_{2}+\cdots+p_{n}$. Suppose that

$$
\operatorname{dim} H^{0}(\theta)-\operatorname{dim} H^{0}(\theta(-D)) \geq n-g+1 .
$$

Then some curve $C \subset S$ of class $\theta$ meets $D$ transversely at $p_{1}, p_{2}, \ldots, p_{n}$.

Proof. Since $p_{1}, p_{2}, \ldots, p_{g}$ are general, and $\left.\theta\right|_{D}=p_{1}+p_{2}+\cdots+p_{n}$, it suffices to show there is a curve of class $\theta$ meeting $D$ dimensionally-transversely and passing through $p_{g+1}, p_{g+2}, \ldots, p_{n}$; the remaining $g$ points of intersection are then forced to be $p_{1}, p_{2}, \ldots, p_{g}$.

For this, we note there is a $\operatorname{dim} H^{0}(\theta)-(n-g)>\operatorname{dim} H^{0}(\theta(-D))$ dimensional space of sections of $\theta$ which vanish at $p_{g+1}, \ldots, p_{n}$. In particular, there is some section which does not vanish along $D$. Its zero locus then gives the required curve $C$. (The curve $C$ meets $D$ dimensionally-transversely, because $C$ does not contain $D$ and $D$ is irreducible.)

Proposition 11.6. Let $C \subset \mathbb{P}^{3}$ be a general $B N$-curve of degree 5 and genus 2 . Then the intersection $C \cap Q$ is a collection of 10 general points lying on a curve of bidegree $(2,2)$ on $Q \simeq \mathbb{P}^{1} \times \mathbb{P}^{1}$. In particular, it is not a collection of 10 general points.

Proof. Since $\operatorname{dim} H^{0}\left(\mathcal{O}_{C}(2)\right)=9$ and $\operatorname{dim} H^{0}\left(\mathcal{O}_{\mathbb{P}^{3}}(2)\right)=10$, we conclude that $C$ lies on a quadric. Restricting to $Q$, we see that $C \cap Q$ lies on a curve of bidegree $(2,2)$.

Conversely, given 10 points $p_{1}, p_{2}, \ldots, p_{10}$ lying on a curve $D$ of bidegree $(2,2)$, we may first find a pair of points $\{x, y\} \subset D$ so that $x+y+2 H \sim p_{1}+\cdots+p_{10}$. We then claim there is a 
smooth quadric containing $D$ and the general 2 -secant line $\overline{x y}$ to $D$. Equivalently, we want to show the map

$$
\{(S, L): L \subset S \supset D\} \mapsto\{L\},
$$

from the space of smooth quadric surfaces $S$ containing $D$ with a choice of line $L$, to the space of 2-secant lines to $D$, is dominant; it suffices to check the vanishing of $H^{1}\left(N_{S}(-D-L)\right.$ ), for any smooth quadric $S$ containing $D$ and line $L$ on $S$, in which lies the obstruction to smoothness of this map. But $N_{S}(-D-L)=\mathcal{O}_{S}(0,-1)$ has no higher cohomology by Kodaira vanishing.

Writing $L \in \operatorname{Pic}(S)$ for the class of the line $\overline{x y}$, we see that $\left.(L+2 H)\right|_{D} \sim p_{1}+\cdots+p_{10}$ as divisor classes. Applying Lemma 11.5, and noting that $\operatorname{dim} H^{0}\left(\mathcal{O}_{S}(2 H+L)\right)=12$ while $\operatorname{dim} H^{0}\left(\mathcal{O}_{S}(L)\right)=2$, there is a curve $C$ of class $2 H+L$ meeting $D$ transversely at $p_{1}, \ldots, p_{10}$. Since $\mathcal{O}_{S}(2 H+L)$ is very ample by inspection, $C$ is smooth (for $p_{1}, \ldots, p_{10}$ general). By results of [8], this implies $C$ is a BN-curve.

Since general points impose independent conditions on the 9-dimensional space of curves of bidegree $(2,2)$, a general collection of 10 points does not lie on a curve of bidegree $(2,2)$. A collection of 10 general points on a general curve of bidegree $(2,2)$ is therefore not a collection of 10 general points.

Proposition 11.7. Let $C \subset \mathbb{P}^{3}$ be a general $B N$-curve of degree 7 and genus 5 . Then the intersection $C \cap Q$ is a collection of 14 points lying on a curve $D \subset Q \simeq \mathbb{P}^{1} \times \mathbb{P}^{1}$, which is general subject to the following conditions:

1. The curve $D$ is of bidegree $(3,3)$.

2. The divisor $C \cap Q-2 H$ on $D$ (where $H$ is the hyperplane class) is effective.

In particular, it is not a collection of 14 general points.

Proof. First we claim the general such curve $C$ lies on a smooth cubic surface $S$ with class $2 H+E_{1}=6 L-E_{1}-2 E_{2}-2 E_{3}-2 E_{4}-2 E_{5}-2 E_{6}$. Indeed, by Lemma 9.3 part 4 , a general curve of this class is smooth and irreducible; such a curve has degree 7 and genus 5 , and in particular is a BN-curve by results of [8]. It remains to see there are no obstructions to lifting a deformation of $C$ to a deformation of the pair $(S, C)$, i.e. that $H^{1}\left(N_{S}(-C)\right)=0$. But $N_{S}(-C)=3 L-2 E_{1}-E_{2}-E_{3}-E_{4}-E_{5}-E_{6}$, which has no higher cohomology by Kodaira vanishing.

Thus, $C \cap Q-2 H$ is the restriction to $D$ of the class of a line on $S$; in particular, $C \cap Q-2 H$ is an effective divisor on $D$.

Conversely, suppose that $p_{1}, p_{2}, \ldots, p_{14}$ are a general collection of 14 points lying on a curve $D$ of bidegree $(3,3)$ with $p_{1}+\cdots+p_{14}-2 H \sim x+y$ effective. We then claim there is a smooth cubic containing $D$ and the general 2 -secant line $\overline{x y}$ to $D$. Equivalently, we want to show the map

$$
\{(S, L): L \subset S \supset D\} \mapsto\{L\}
$$

from the space of smooth cubic surfaces $S$ containing $D$ with a choice of line $L$, to the space of 2-secant lines to $D$, is dominant; for this it suffices to check the vanishing of $H^{1}\left(N_{S}(-D-L)\right)$. But $N_{S}(-D-L)=3 L-2 E_{1}-E_{2}-E_{3}-E_{4}-E_{5}-E_{6}$, which has no higher cohomology by Kodaira vanishing. 
Choosing an isomorphism $S \simeq \mathrm{Bl}_{\Gamma} \mathbb{P}^{2}$ where $\Gamma=\left\{q_{1}, q_{2}, \ldots, q_{6}\right\}$, so that the line $\overline{x y}=E_{1}$ is the exceptional divisor over $q_{1}$, we now look for a curve $C \subset S$ of class

$$
[C]=6 L-E_{1}-2 E_{2}-2 E_{3}-2 E_{4}-2 E_{5}-2 E_{6}
$$

Again by Lemma 9.3, the general such curve is smooth and irreducible; such a curve has degree 7 and genus 5 , and in particular is a BN-curve by results of [8]. Note that

$$
\operatorname{dim} H^{0}\left(\mathcal{O}_{S}\left(6 L-E_{1}-2 E_{2}-2 E_{3}-2 E_{4}-2 E_{5}-2 E_{6}\right)\right)=12 \quad \text { and } \quad \operatorname{dim} H^{0}\left(\mathcal{O}_{S}\left(E_{1}\right)\right)=1 .
$$

Applying Lemma 11.5, we conclude that some curve of our given class meets $D$ transversely at $p_{1}, p_{2}, \ldots, p_{14}$, as desired.

It remains to see from this description that $C \cap Q$ is not a general collection of 14 points. For this, first note that there is a 15-dimensional space of such curves $D\left(\right.$ as $\left.\operatorname{dim} H^{0}\left(\mathcal{O}_{Q}(3,3)\right)=16\right)$. On each each curve, there is a 2-dimensional family of effective divisors $\Delta$; and for fixed $\Delta$, a 10 dimensional family of divisors linearly equivalent to $2 H+\Delta$ (because $\operatorname{dim} H^{0}\left(\mathcal{O}_{D}(2 H+\Delta)\right)=11$ by Riemann-Roch). Putting this together, there is an (at most) $15+2+10=27$-dimensional family of such collections of points. But $\operatorname{Sym}^{14}(Q)$ has dimension 28. In particular, collections of such points cannot be general.

Proposition 11.8. Let $C \subset \mathbb{P}^{3}$ be a general $B N$-curve of degree 8 and genus 6 . Then the intersection $C \cap Q$ is a general collection of 16 points on a curve of bidegree $(3,3)$ on $Q \simeq \mathbb{P}^{1} \times \mathbb{P}^{1}$. In particular, it is not a collection of 16 general points.

Proof. Since $\operatorname{dim} H^{0}\left(\mathcal{O}_{C}(3)\right)=19$ and $\operatorname{dim} H^{0}\left(\mathcal{O}_{\mathbb{P}^{3}}(3)\right)=20$, we conclude that $C$ lies a cubic surface. Restricting this cubic to $Q$, we see that $C \cap Q$ lies on a curve of bidegree $(3,3)$.

Conversely, take a general collection $p_{1}, \ldots, p_{16}$ of 16 points on a curve $D$ of bidegree $(3,3)$. The divisor $p_{1}+\cdots+p_{16}-2 H$ is of degree 4 on a curve $D$ of genus 4 ; it is therefore effective, say

$$
p_{1}+\cdots+p_{16}-2 H \sim x+y+z+w .
$$

We then claim there is a smooth cubic containing $D$ and the general 2-secant lines $\overline{x y}$ and $\overline{z w}$ to $D$. Equivalently, we want to show the map

$$
\left\{\left(S, E_{1}, E_{2}\right): E_{1}, E_{2} \subset S \supset D\right\} \mapsto\left\{\left(E_{1}, E_{2}\right)\right\}
$$

from the space of smooth cubic surfaces $S$ containing $D$ with a choice of pair of disjoint lines $\left(E_{1}, E_{2}\right)$, to the space of pairs of 2-secant lines to $D$, is dominant; for this it suffices to check the vanishing of $H^{1}\left(N_{S}\left(-D-E_{1}-E_{2}\right)\right)$. But $N_{S}\left(-D-E_{1}-E_{2}\right)=3 L-2 E_{1}-2 E_{2}-E_{3}-E_{4}-E_{5}-E_{6}$, which has no higher cohomology by Kawamata-Viehweg vanishing.

We now look for a curve $C \subset S$ of class

$$
[C]=6 L-E_{1}-E_{2}-2 E_{3}-2 E_{4}-2 E_{5}-2 E_{6},
$$

which is of degree 8 and genus 6. By Lemma 9.3, we conclude that $C$ is smooth and irreducible; by results of [8], this implies the general curve of this class is a $\mathrm{BN}$-curve. Note that

$\operatorname{dim} H^{0}\left(\mathcal{O}_{S}\left(6 L-E_{1}-E_{2}-2 E_{3}-2 E_{4}-2 E_{5}-2 E_{6}\right)\right)=14 \quad$ and $\quad \operatorname{dim} H^{0}\left(\mathcal{O}_{S}\left(E_{1}+E_{2}\right)\right)=1$. 
Applying Lemma 11.5, we conclude that some curve of our given class meets $D$ transversely at $p_{1}, p_{2}, \ldots, p_{16}$, as desired.

Since general points impose independent conditions on the 16-dimensional space of curves of bidegree $(3,3)$, a general collection of 16 points will not lie any curve of bidegree $(3,3)$. Our collection of points is therefore not general.

Proposition 11.9. Let $C \subset \mathbb{P}^{4}$ be a general $B N$-curve of degree 9 and genus 6 . Then the intersection $C \cap H$ is a general collection of 9 points on an elliptic normal curve in $H \simeq \mathbb{P}^{3}$. In particular, it is not a collection of 9 general points.

Proof. Since $\operatorname{dim} H^{0}\left(\mathcal{O}_{C}(2)\right)=13$ and $\operatorname{dim} H^{0}\left(\mathcal{O}_{\mathbb{P}^{4}}(2)\right)=15$, we conclude that $C$ lies on the intersection of two quadrics. Restricting these quadrics to $H \simeq \mathbb{P}^{3}$, we see that $C \cap H$ lies on the intersection of two quadrics, which is an elliptic normal curve.

Conversely, let $p_{1}, p_{2}, \ldots, p_{9}$ be a collection of 9 points lying on an elliptic normal curve $D \subset \mathbb{P}^{3}$. Since $D$ is an elliptic curve, there exists (a unique) $x \in D$ with

$$
\mathcal{O}_{D}\left(p_{1}+\cdots+p_{9}\right)(-2) \simeq \mathcal{O}_{D}(x) .
$$

Let $M$ be a general line through $x$. We then claim there is a quartic Del Pezzo surface containing $D$ and the general 1-secant line $M$. Equivalently, we want to show the map

$$
\left\{\left(S, E_{1}\right): E_{1} \subset S \supset D\right\} \mapsto\left\{E_{1}\right\},
$$

from the space of smooth Del Pezzo surfaces $S$ containing $D$ with a choice of line $E_{1}$, to the space of 1-secant lines to $D$, is dominant; for this it suffices to check the vanishing of $H^{1}\left(N_{S}\left(-D-E_{1}\right)\right.$ ). But $N_{S}\left(-D-E_{1}\right)$ is a direct sum of two copies of the line bundle $3 L-2 E_{1}-E_{2}-E_{3}-E_{4}-E_{5}$, which has no higher cohomology by Kodaira vanishing.

We now consider curves $C \subset S$ of class

$$
[C]=6 L-E_{1}-2 E_{2}-2 E_{3}-2 E_{4}-2 E_{5},
$$

which are of degree 9 and genus 6 . By Lemma 9.6, we conclude that $C$ is smooth and irreducible; by results of [7], this implies the general curve of this class is a BN-curve. Note that

$\operatorname{dim} H^{0}\left(\mathcal{O}_{S}\left(6 L-E_{1}-2 E_{2}-2 E_{3}-2 E_{4}-2 E_{5}\right)\right)=15$ and $\operatorname{dim} H^{0}\left(\mathcal{O}_{S}\left(3 L-E_{2}-E_{3}-E_{4}-E_{5}\right)\right)=6$.

Applying Lemma 11.5, we conclude that some curve of our given class meets $D$ transversely at $p_{1}, p_{2}, \ldots, p_{9}$, as desired.

By Corollary 1.4 of [2], there does not exist an elliptic normal curve in $\mathbb{P}^{3}$ passing through 9 general points.

\subsection{The Final Two Exceptional Cases}

We have exactly two remaining exceptional cases: The intersection of a general BN-curve of degree 6 and genus 2 in $\mathbb{P}^{3}$ with a quadric, and the intersection of a general BN-curve of degree 10 and genus 7 in $\mathbb{P}^{4}$ with a hyperplane. We will show in the first case that the intersection fails to be general since $C$ is the projection of a curve $\widetilde{C} \subset \mathbb{P}^{4}$, where $\widetilde{C}$ lies on a surface of small degree (a cubic scroll). In the second case, the intersection fails to be general since $C$ is contained in a quadric hypersurface. 
Proposition 11.10. Let $C \subset \mathbb{P}^{3}$ be a general $B N$-curve of degree 6 and genus 2 . Then the intersection $C \cap Q$ is a collection of 12 points lying on a curve $D \subset Q \simeq \mathbb{P}^{1} \times \mathbb{P}^{1}$, which is general subject to the following conditions:

1. The curve $D$ is of bidegree $(3,3)$ (and so is in particular of arithmetic genus 4 ).

2. The curve $D$ has two nodes (and so is in particular of geometric genus 2).

3. The divisors $\mathcal{O}_{D}(2,2)$ and $C \cap D$ are linearly equivalent when pulled back to the normalization of $D$.

In particular, it is not a collection of 12 general points.

Proof. We first observe that $\operatorname{dim} H^{0}\left(\mathcal{O}_{C}(1)\right)=5$, so $C$ is the projection from a point $p \in \mathbb{P}^{4}$ of a curve $\widetilde{C} \subset \mathbb{P}^{4}$ of degree 6 and genus 2 . Write $\pi: \mathbb{P}^{4} \rightarrow \mathbb{P}^{3}$ for the map of projection from $p$, and define the quadric hypersurface $\widetilde{Q}=\pi^{-1}(Q)$.

Let $S \subset \mathbb{P}^{4}$ be the surface swept out by joining pairs of points on $\widetilde{C}$ conjugate under the hyperelliptic involution. By Corollary 13.3 of [2], $S$ is a cubic surface; in particular, since $S$ has a ruling, $S$ is a cubic scroll. Write $H$ for the hyperplane section on $S$, and $F$ for the class of a line of the ruling.

Th curve $\widetilde{D}=\widetilde{Q} \cap S$ (which for $C$ general is smooth by Kleiman transversality), is of degree 6 and genus 2. By construction, the intersection $C \cap Q$ lies on $D=\pi(\widetilde{D})$. Since $D=\pi(S) \cap Q$, it is evidently a curve of bidegree $(3,3)$ on $Q \simeq \mathbb{P}^{1} \times \mathbb{P}^{1}$. Moreover, since $\widetilde{D}$ has genus 2 , the geometric genus of $D$ is 2 . In particular, $D$ has two nodes.

Next, we note that on $S$, the curve $\widetilde{C}$ has class $2 H$. Indeed, if $[\widetilde{C}]=a \cdot H+b \cdot F$, then $a=\widetilde{C} \cdot F=2$ and $3 a+b=\widetilde{C} \cdot H=6$; solving for $a$ and $b$, we obtain $a=2$ and $b=0$. Consequently, $\widetilde{C} \cap \widetilde{D}$ has class $2 H$ on $\widetilde{D}$. Or equivalently, $C \cap D=\pi(\widetilde{C} \cap \widetilde{D})$ has class equal to $\mathcal{O}_{D}(2)=\mathcal{O}_{D}(2,2)$ when pulled back to the normalization.

Conversely, take 12 points on $D$ satisfying our assumptions. Write $\widetilde{D}$ for the normalization of $D$, and $p_{1}, p_{2}, \ldots, p_{12}$ for the preimages of our points in $\widetilde{D}$. We begin by noting that $\operatorname{dim} H^{0}\left(\mathcal{O}_{\widetilde{D}}(1)\right)=5$, so $D$ is the projection from a point $p \in \mathbb{P}^{4}$ of $\widetilde{D} \subset \mathbb{P}^{4}$ of degree 6 and genus 2. As before, write $\pi: \mathbb{P}^{4} \rightarrow \mathbb{P}^{3}$ for the map of projection from $p$, and define the quadric hypersurface $\widetilde{Q}=\pi^{-1}(Q)$.

Again, we let $S \subset \mathbb{P}^{4}$ be the surface swept out by joining pairs of points on $\widetilde{D}$ conjugate under the hyperelliptic involution. As before, $S$ is a cubic scroll; write $H$ for the hyperplane section on $S$, and $F$ for the class of a line of the ruling. Note that $\widetilde{D} \subseteq \widetilde{Q} \cap S$; and since both sides are curves of degree 6 , we have $\widetilde{D}=\widetilde{Q} \cap S$.

It now suffices to find a curve $\widetilde{C} \subset S$ of class $2 H$, meeting $\widetilde{D}$ transversely in $p_{1}, \ldots, p_{12}$. For this, note that

$$
\operatorname{dim} H^{0}\left(\mathcal{O}_{S}(2 H)\right)=12 \quad \text { and } \quad \operatorname{dim} H^{0}\left(\mathcal{O}_{S}\right)=1 .
$$

Applying Lemma 11.5 yields the desired conclusion.

It remains to see from this description that $C \cap Q$ is not a general collection of 12 points. For this, we first note that such a curve $D \subset \mathbb{P}^{1} \times \mathbb{P}^{1}$ is the same as specifying an abstract curve of genus 2, two lines bundles of degree 3 (corresponding to the pullbacks of $\mathcal{O}_{\mathbb{P}^{1}}(1)$ from each factor), and a basis-up-to-scaling for their space of sections (giving us two maps 
$\left.D \rightarrow \mathbb{P}^{1}\right)$. Since there is a 3 -dimensional moduli space of abstract curves $D$ of genus 2 , and $\operatorname{dim} \operatorname{Pic}^{3}(D)=2$, and there is a 3-dimensional family of bases-up-to-scaling of a 2-dimensional vector space, the dimension of the space of such curves $D$ is $3+2+2+3+3=13$. Our condition $p_{1}+\cdots+p_{12} \sim 2 H$ then implies collections of such points on a fixed $D$ are in bijection with elements of $\mathbb{P O}_{D}(2 H) \simeq \mathbb{P}^{10}$. Putting this together, there is an (at most) $13+10=23$ dimensional family of such collections of points. But $\operatorname{Sym}^{12}(Q)$ has dimension 24. In particular, collections of such points cannot be general.

Proposition 11.11. Let $C \subset \mathbb{P}^{4}$ be a general $B N$-curve of degree 10 and genus 7 . Then the intersection $C \cap H$ is a general collection of 10 points on a quadric in $H \simeq \mathbb{P}^{3}$. In particular, it is not a collection of 10 general points.

Proof. Since $\operatorname{dim} H^{0}\left(\mathcal{O}_{C}(2)\right)=14$ and $\operatorname{dim} H^{0}\left(\mathcal{O}_{\mathbb{P}^{4}}(2)\right)=15$, we conclude that $C$ lies on a quadric. Restricting this quadric to $H \simeq \mathbb{P}^{3}$, we see that $C \cap H$ lies on a quadric.

For the converse, we take general points $p_{1}, \ldots, p_{10}$ lying on a general (thus smooth) quadric $Q$. Since $\operatorname{dim} H^{0}\left(\mathcal{O}_{Q}(3,3)\right)=16$, we may find a curve $D \subset Q$ of type $(3,3)$ passing through $p_{1}, \ldots, p_{10}$. As divisor classes on $D$, suppose that

$$
p_{1}+p_{2}+\cdots+p_{10}-H \sim x+y+z+w
$$

We now pick a general (quartic) rational normal curve $R \subset \mathbb{P}^{4}$ whose hyperplane section is $\{x, y, z, w\}$.

We then claim there is a smooth sextic K3 surface $S \subset \mathbb{P}^{4}$ containing $D$ and the general 2 -secant lines $\overline{x y}$ and $\overline{z w}$ to $D$. Equivalently, we want to show the map

$$
\{(S, R): R \subset S\} \mapsto\{(R, D)\}
$$

from the space of smooth sextic K3 surfaces $S$, to the space of pairs $(R, D)$ where $R$ is a rational normal curve meeting the canonical curve $D=S \cap H$ in four points, is dominant; for this it suffices to check the vanishing of $H^{1}\left(N_{S}(-H-R)\right)$ at any smooth sextic K3 containing a rational normal curve $R$ (where $H=[D]$ is the hyperplane class on $S$ ). We first note that a sextic K3 surface $S$ containing a rational normal curve $R$ exists, by Theorem 1.1 of [9]. On this K3 surface, our vector bundle $N_{S}(-H-R)$ is the direct sum of the line bundles $H-R$ and $2 H-R$; consequently, it suffices to show $H^{1}\left(\mathcal{O}_{S}(n)(-R)\right)=0$ for $n \geq 1$. For this we use the exact sequence

$$
\left.0 \rightarrow \mathcal{O}_{S}(n)(-R) \rightarrow \mathcal{O}_{S}(n) \rightarrow \mathcal{O}_{S}(n)\right|_{R}=\mathcal{O}_{R}(n) \rightarrow 0
$$

and note that $H^{1}\left(\mathcal{O}_{S}(n)\right)=0$ by Kodaira vanishing, while $H^{0}\left(\mathcal{O}_{S}(n)\right) \rightarrow H^{0}\left(\mathcal{O}_{R}(n)\right)$ is surjective since $R$ is projectively normal. This shows the existence of the desired K3 surface $S$ containing $D$ and the general 4-secant rational normal curve $R$.

Next, we claim that the linear series $H+R$ on $S$ is basepoint-free. To see this, we first note that $H$ is basepoint free, so any basepoints must lie on the curve $R$. Now the short exact sequence of sheaves

$$
\left.0 \rightarrow \mathcal{O}_{S}(H) \rightarrow \mathcal{O}_{S}(H+R) \rightarrow \mathcal{O}_{S}(H+R)\right|_{R} \rightarrow 0
$$


gives a long exact sequence in cohomology

$$
\cdots \rightarrow H^{0}\left(\mathcal{O}_{S}(H+R)\right) \rightarrow H^{0}\left(\left.\mathcal{O}_{S}(H+R)\right|_{R}\right) \rightarrow H^{1}\left(\mathcal{O}_{S}(H)\right) \rightarrow \cdots
$$

Since the complete linear series attached to $\left.\mathcal{O}_{S}(H+R)\right|_{R} \simeq \mathcal{O}_{\mathbb{P}^{1}}(2)$ is basepoint-free, it suffices to show that $H^{0}\left(\mathcal{O}_{S}(H+R)\right) \rightarrow H^{0}\left(\left.\mathcal{O}_{S}(H+R)\right|_{R}\right)$ is surjective. For this, it suffices to note that $H^{1}\left(\mathcal{O}_{S}(H)\right)=0$ by Kodaira vanishing.

Thus, $H+R$ is basepoint-free. In particular, the Bertini theorem implies the general curve of class $H+R$ is smooth. Such a curve is of degree 10 and genus 7; in particular it is a BN-curve by results of [7]. So it suffices to find a curve of class $H+R$ on $S$ passing through $p_{1}, p_{2}, \ldots, p_{10}$. By construction, as divisors on $D$, we have

$$
p_{1}+p_{2}+\cdots+p_{10} \sim H+R
$$

By Lemma 11.5, it suffices to show $\operatorname{dim} H^{0}\left(\mathcal{O}_{S}(H+R)\right)=8$ and $\operatorname{dim} H^{0}\left(\mathcal{O}_{S}(R)\right)=1$.

More generally, for any smooth curve $X \subset S$ of genus $g$, we claim $\operatorname{dim} H^{0}\left(\mathcal{O}_{S}(X)\right)=1+g$. To see this, we use the exact sequence

$$
\left.0 \rightarrow \mathcal{O}_{S} \rightarrow \mathcal{O}_{S}(X) \rightarrow \mathcal{O}_{S}(X)\right|_{X} \rightarrow 0
$$

which gives rise to a long exact sequence in cohomology

$$
0 \rightarrow H^{0}\left(\mathcal{O}_{S}\right) \rightarrow H^{0}\left(\mathcal{O}_{S}(X)\right) \rightarrow H^{0}\left(\left.\mathcal{O}_{S}(X)\right|_{X}\right) \rightarrow H^{1}\left(\mathcal{O}_{S}\right) \rightarrow \cdots .
$$

Because $H^{1}\left(\mathcal{O}_{S}\right)=0$, we thus have

$$
\begin{aligned}
\operatorname{dim} H^{0}\left(\mathcal{O}_{S}(X)\right) & =\operatorname{dim} H^{0}\left(\left.\mathcal{O}_{S}(X)\right|_{X}\right)+\operatorname{dim} H^{0}\left(\mathcal{O}_{S}\right) \\
& =\operatorname{dim} H^{0}\left(\left.K_{S}(X)\right|_{X}\right)+1 \\
& =\operatorname{dim} H^{0}\left(K_{X}\right)+1 \\
& =g+1 .
\end{aligned}
$$

In particular, $\operatorname{dim} H^{0}\left(\mathcal{O}_{S}(H+R)\right)=8$ and $\operatorname{dim} H^{0}\left(\mathcal{O}_{S}(R)\right)=1$, as desired.

Since general points impose independent conditions on the 10-dimensional space of quadrics, a general collection of 10 points will not lie on a quadric. In particular, our hyperplane section here is not a general collection of 10 points.

\section{References}

[1] Atanas Atanasov. Interpolation and vector bundles on curves. Available at http://arxiv.org/abs/1404.4892.

[2] Atanas Atanasov, Eric Larson, and David Yang. Interpolation for normal bundles of general curves. Available at http://arxiv.org/abs/1509.01724v2.

[3] Edoardo Ballico and Philippe Ellia. Beyond the maximal rank conjecture for curves in $\mathbb{P}^{3}$. In Space curves (Rocca di Papa, 1985), volume 1266 of Lecture Notes in Math., pages 1-23. Springer, Berlin, 1987. 
[4] Geir Ellingsrud and André Hirschowitz. Sur le fibré normal des courbes gauches. C. R. Acad. Sci. Paris Sér. I Math., 299(7):245-248, 1984.

[5] R. Hartshorne and A. Hirschowitz. Smoothing algebraic space curves. In Algebraic geometry, Sitges (Barcelona), 1983, volume 1124 of Lecture Notes in Math., pages 98-131. Springer, Berlin, 1985.

[6] A. Hirschowitz. Sur la postulation générique des courbes rationnelles. Acta Math., 146(34):209-230, 1981.

[7] Hristo Iliev. On the irreducibility of the Hilbert scheme of space curves. Proc. Amer. Math. Soc., 134(10):2823-2832 (electronic), 2006.

[8] C. Keem and SeonJa Kim. Irreducibility of a subscheme of the Hilbert scheme of complex space curves. J. Algebra, 145(1):240-248, 1992.

[9] Andreas Leopold Knutsen. Smooth curves on projective K3 surfaces. Math. Scand., 90(2):215-231, 2002.

[10] Eric Larson. Constructing reducible brill-noether curves. Available at http://arxiv.org/abs/1603.02301.

[11] Eric Larson. Degenerations of curves in projective space and the maximal rank conjecture. https://arxiv.org/abs/1809.05980.

[12] Eric Larson. The maximal rank conjecture. https://arxiv.org/abs/1711.04906.

[13] Daniel Perrin. Courbes passant par $m$ points généraux de $\mathbf{P}^{3}$. Mém. Soc. Math. France (N.S.), (28-29):138, 1987.

[14] Michel Raynaud. Sections des fibrés vectoriels sur une courbe. Bull. Soc. Math. France, 110(1):103-125, 1982. 